\title{
Mathematical modelling of the phloem: the importance of diffusion on sugar transport at osmotic equilibrium
}

\author{
S. Payvandi ${ }^{a, b}$, K.R. Daly ${ }^{a, b}$, K.C. Zygalakis ${ }^{c, b}$, T. Roose ${ }^{a, b}$
}

$a$ : Engineering Sciences, Faculty of Engineering and the Environment, University of Southampton, University Road, Southampton, SO17 1BJ,

UK

b: Crop Systems Engineering Group, Institute for Life Sciences, University of Southampton, University Road, Southampton, SO17 1BJ, UK $c$ : Mathematical Sciences, Faculty of Social and Human Sciences, University of Southampton, University Road, Southampton, SO17 1DN,

UK

\begin{abstract}
Plants rely on the conducting vessels of the phloem to transport the products of photosynthesis from the leaves to the roots, or to any other organs, for growth, metabolism and storage. Transport within the phloem is due to an osmotically-generated pressure gradient and is hence inherently nonlinear. Since convection dominates over diffusion in the main bulk flow, the effects of diffusive transport have generally been neglected by previous authors. However, diffusion is important due to boundary layers that form at the ends of the phloem, and at the leaf-stem and stem-root boundaries. We present a mathematical model of transport which includes the effects of diffusion. We solve the system analytically in the limit of high Munch number which corresponds to osmotic equilibrium, and numerically for all parameter values. We find that the bulk solution is dependent on the diffusion-dominated boundary layers. Hence, even for large Péclet number, it is not always correct to neglect diffusion. We consider the cases of passive and active sugar loading and unloading. We show that for active unloading the solutions diverge with increasing Péclet. For passive unloading the convergence of the solutions is dependent on the magnitude of loading. Diffusion also permits the modelling of an axial efflux of sugar in the root zone which may be important for the growing root tip and for promoting symbiotic biological interactions in the soil. Therefore, diffusion is an essential mechanism for transport in the phloem and must be included to accurately predict flow.
\end{abstract}




\section{Introduction}

The vascular transport systems are essential to the functioning of plants. Whilst the xylem vessels are responsible for transporting water and nutrients derived from the soil, the phloem is responsible for transporting sugars produced in the leaves to organs that need sugar, such as the roots. Understanding phloem transport is essential for understanding plant functioning, and may lead to advances in improving plant growth. In the agricultural industry, foliar-applied fertilisers are being considered as a way to improve the efficiency of fertiliser use (Fernández \& Brown, 2013), and the optimal application of these fertilisers may depend on the dynamics of phloem transport.

In contrast to the lignified xylem, the phloem is comprised of living tissue consisting of sieve elements and companion cells (Van Bel, 2003). The sieve elements form conducting segments, which are separated from each other by sieve plates (porous structures that become blocked at times of injury). Sugars are transported within the phloem as sap, which consists of water, dissolved sugars and minerals. The transport of sap is pressure driven, but the pressure gradient is osmotically generated. At the leaves, sugars are actively loaded into the sieve cells which decreases the osmotic potential and hence the water potential. This causes water to enter the phloem from surrounding tissues and consequently raises the hydrostatic pressure. At the sink tissues, unloading of sugars, for use in growth, metabolism or storage, increases the osmotic potential (increasing the water potential) which causes water to leave the phloem sap, decreasing the hydrostatic pressure. Therefore, due to the activities of the leaves and the roots, a pressure gradient is maintained which causes the flow of fluid. This process, known as osmotically generated pressure driven flow was proposed by Münch (1926), and has come to be the accepted mechanism of sap flow (Thompson \& Holbrook, 2003a). The Münchian flow of sap is inherently nonlinear since sugar is advected from the source to sink tissue by the flow of sap, but the flow itself is set up by the sugar concentration gradient. This nonlinearity has made analysis of the phloem difficult. In addition, the precise details of loading and unloading processes are still unknown and there is much research into whether loading or unloading dominates the flow (Patrick, 1997; Turgeon, 2010; De Schepper et al., 2013).

The majority of phloem modelling has been numerical, with the most complete study being conducted by Thompson \& Holbrook (2003a) who considered time-dependent phloem flow with a nonlinear osmotic potential, nonlinear viscosity, elastic walls, the effects of sieve plates, and loading and unloading. They also provided a comprehensive review of other phloem models (see appendix in Thompson \& Holbrook (2003a)). They extended their model in Thompson \& Holbrook (2003b, 2004), and Thompson (2005) to consider phloem flow under the state of osmotic equilibrium and to in- 
vestigate the propagation of concentration and pressure waves down the phloem. Jensen et al. $(2009,2011,2012)$ followed the approach of Thompson \& Holbrook (2003a,b, 2004) modelling the phloem flow analytically and numerically. They considered transport in the loading, unloading, and translocation zones, first analysing explicit loading and then implicit loading. Note that the translocation zone denotes the region where sugar transport is due to Münchian flow, resulting in predominantly axial transport of sugars. The translocation zone usually refers to the stem of the plant. This is in contrast to the loading and unloading zones of the leaves and the roots where large radial transport of sugars occurs.

Due to the large magnitude of the Péclet number (Thompson \& Holbrook, 2003a), convection of sugar dominates over diffusion. This has led to the majority of previous authors neglecting the diffusive terms entirely in their analysis. Whilst this assumption may be valid in the bulk of the phloem, it is possible that due to the nonlinearity of the flow, boundary layer effects at the ends of the phloem may become significant and influence the bulk flow. Therefore, it is our aim to use matched asymptotic analysis to determine whether neglecting diffusion entirely is a valid assumption. The application of matched asymptotics to flow and transport in the phloem is limited to the work of Pickard \& Abraham-Shrauner (2009). These authors analytically considered phloem flow including diffusion. However, their analysis only considered the translocation zone and neglected longitudinal variation of pressure. These assumptions ensure that velocity is driven solely by the concentration gradient, and neglects the end-effects caused by the root and leaf boundaries, which may significantly influence flow.

In this paper we follow the approaches of Jensen et al. (2011, 2012) and consider the flow within the loading and unloading zones as well as the translocation zone. For simplicity we do not consider a nonlinear viscosity or a nonlinear osmotic potential, but instead investigate the effect of diffusion on flow in the phloem for biologically-relevant parameters, i.e., when diffusion within the main bulk of the phloem is low, and when the system is close to osmotic equilibrium.

In Section 2 we present the model which describes the transport of water and sugar including the effects of diffusion. The majority of previous phloem modelling has been concerned with transport in trees, and as a representative example of a tree, we consider the model parameters for black locust in Section 3. We also consider the model parameters for wheat to represent transport in cereals. We then nondimensionalise the model, and in Section 4 we present the analytical method and solution to the model. In Section 5 we compare the analytical solution to the numerical solution for biologically-relevant parameters and investigate the effect that the main model parameters have on the flow. In particular, we investigate the effect of diffusion on the phloem flow and find that only for certain parameter values do the solutions become independent of Péclet number such that diffusion 
can be neglected. Finally, in Section 6 we discuss the implications of the model findings.

\section{Model}

We consider the transport of water and photosynthates from leaves to roots in the phloem vessels of plants. We follow a similar approach to the phloem model of Jensen et al. (2011, 2012) and the xylem model of Payvandi et al. (2014), and separate the plant and phloem tissue into three functional zones in the $\hat{z}$ direction; the leaves $L$, the stem $S$, and the roots $R$ as shown in Figure 1 in Section 4 . The leaf zone is represented by $0<\hat{z}<\hat{z}_{1}$, the stem zone is $\hat{z}_{1}<\hat{z}<\hat{z}_{2}$ and the root zone is $\hat{z}_{2}<\hat{z}<\hat{z}_{3}$ where $\hat{z}, \hat{z}_{1}, \hat{z}_{2}$ and $\hat{z}_{3}$ are measured in metres. All symbols are given in Table 1. In Section 3, we will show that the model parameters are highly dependent on phloem radius and plant length $\hat{z}_{3}$. Therefore we consider two different-sized plants: the crop plant Triticum aestivum (wheat) and the tall tree Robinia pseudoacacia (black locust). Parameter values for these two plants are given in Table 1.

Multiple products of photosynthesis are found in the phloem (Barbaroux et al., 2003), but for simplicity we group these sugar-containing compounds into one variable $\hat{c}\left(\mathrm{~mol} \mathrm{~m}^{-3}\right)$. Whilst other nutrients such as potassium are also transported in the phloem, in this paper we assume that the osmotic potential gradient is created by sugar, $\hat{c}$, only.

\subsection{Water transport in the phloem}

We consider the flow within a single phloem vessel since transport along all the vessels is summative. The axial flow along the phloem vessel is governed by Poiseuille's law (Thompson \& Holbrook (2003a)), such that the dimensional axial flux, $\hat{q}_{z}^{i}\left(\mathrm{~m}^{3} \mathrm{~s}^{-1}\right)$, is given by

$$
\hat{q}_{z}^{i}=-\hat{k}_{z}\left(\frac{d \hat{p}^{i}}{d \hat{z}}\right), \quad \hat{k}_{z}=\frac{\pi \hat{a}^{4}}{8 \hat{\mu}}
$$

where $i$ is an index for the plant zone; $i=L, S, R$, refers to the leaf, stem, and root zones respectively. $\hat{k}_{z}$ is the phloem axial conductivity $\left(\mathrm{m}^{4} \mathrm{~Pa}^{-1} \mathrm{~s}^{-1}\right), \hat{p}^{i}$ is the internal fluid pressure $(\mathrm{Pa})$ in the phloem vessel, $\hat{a}$ is the radius $(\mathrm{m})$ of the phloem vessel, and $\hat{\mu}$ is the viscosity (Pas) of the phloem sap. The radial flux per unit area $\hat{q}_{r}^{i}\left(\mathrm{~m} \mathrm{~s}^{-1}\right)$ into the vessel is given by the difference in water potential $(\mathrm{Pa})$ between the inside of the phloem vessel, $\hat{\Psi}^{i}$, and the surrounding apoplasm, $\hat{\Psi}_{e x t}$,

$$
\hat{q}_{r}^{i}=\hat{L}_{p}\left(\hat{\Psi}_{e x t}-\hat{\Psi}^{i}\right),
$$

where $\hat{L}_{p}\left(\mathrm{~m} \mathrm{~Pa}^{-1} \mathrm{~s}^{-1}\right)$ is the hydraulic conductivity of the phloem boundary. We assume that the water potential outside the phloem is constant and 


\begin{tabular}{|c|c|c|c|c|}
\hline Symbol & Description & Value & Units & Source \\
\hline$\overline{L L, S, R}$ & 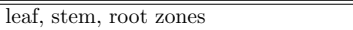 & "- & ב- & "ב- \\
\hline$i$ & zone index & - & - & - \\
\hline$\hat{a}$ & phloem vessel radius & $\begin{array}{l}6 \times 10^{-6} \\
10 \times 10^{-6}\end{array}$ & $\begin{array}{l}\mathrm{m} \\
\mathrm{m}\end{array}$ & $\begin{array}{l}\text { Wheat, Thompson \& Holbrook (2003b) } \\
\text { Black locust, Thompson \& Holbrook (2004) }\end{array}$ \\
\hline$\hat{z}_{1}$ & position of leaf-stem boundary & $\begin{array}{l}0.23 \\
10\end{array}$ & $\begin{array}{l}\mathrm{m} \\
\mathrm{m}\end{array}$ & $\begin{array}{l}\text { Wheat Kutschera et al. (2009) } \\
\text { Black locust, Section } 3\end{array}$ \\
\hline$\hat{z}_{2}$ & position of stem-root boundary & $\begin{array}{l}0.33 \\
40\end{array}$ & $\begin{array}{l}\mathrm{m} \\
\mathrm{m}\end{array}$ & $\begin{array}{l}\text { Wheat, Kutschera et al. (2009) } \\
\text { Black locust, Jensen et al. (2011) }\end{array}$ \\
\hline$\hat{\hat{z}_{3}}$ & length of plant & $\begin{array}{l}0.81 \\
50\end{array}$ & $\begin{array}{l}\mathrm{m} \\
\mathrm{m}\end{array}$ & $\begin{array}{l}\text { Wheat, Kutschera et al. (2009) } \\
\text { Black locust, Section } 3\end{array}$ \\
\hline$\hat{C}$ & typical concentration & $\begin{array}{l}877 \\
463.23\end{array}$ & $\begin{array}{l}\mathrm{mol} \mathrm{m}^{-3} \\
\mathrm{~mol} \mathrm{~m}^{-3}\end{array}$ & $\begin{array}{l}\text { Wheat, Thompson \& Holbrook (2003b) } \\
\text { Black locust, Thompson \& Holbrook (2004) }\end{array}$ \\
\hline$\hat{P}$ & pressure scale & $\begin{array}{l}2.25 \times 10^{4} \\
5 \times 10^{5}\end{array}$ & $\begin{array}{l}\mathrm{Pa} \\
\mathrm{Pa}\end{array}$ & $\begin{array}{l}\text { Wheat, Calculated using } \hat{P}=\hat{z}_{3} \hat{\mu} \hat{U} / \hat{a}^{2} \\
\text { Black locust, Calculated using } \hat{P}=\hat{z}_{3} \hat{\mu} \hat{U} / \hat{a}^{2}\end{array}$ \\
\hline$\hat{k}_{z}$ & phloem axial conductivity & $\begin{array}{l}1.02 \times 10^{-19} \\
7.85 \times 10^{-19}\end{array}$ & $\begin{array}{l}\mathrm{m}^{4} \mathrm{~Pa}^{-1} \mathrm{~s}^{-1} \\
\mathrm{~m}^{4} \mathrm{~Pa}^{-1} \mathrm{~s}^{-1}\end{array}$ & $\begin{array}{l}\text { Wheat, Calculated using } \hat{k}_{z}=\frac{\pi \hat{a}^{4}}{8 \hat{\mu}} \\
\text { Black locust, Calculated using } \hat{k}_{z}=\frac{\pi \hat{a}^{4}}{8 \hat{\mu}}\end{array}$ \\
\hline$\hat{U}$ & typical sap velocity & $2 \times 10^{-4}$ & $\mathrm{~ms}^{-1}$ & Section 3 \\
\hline$\hat{L}_{p}$ & hydraulic conductivity of membrane & $5 \times 10^{-14}$ & $\mathrm{mPa}^{-1} \mathrm{~s}^{-1}$ & Thompson \& Holbrook (2003a) \\
\hline$\hat{D}$ & diffusivity of sugar in water & $5 \times 10^{-10}$ & $\mathrm{~m}^{2} \mathrm{~s}^{-1}$ & Thompson \& Holbrook (2003a) \\
\hline$\hat{\mu}$ & fluid viscosity in phloem & $5 \times 10^{-3}$ & $\mathrm{Pas}$ & Jensen et al. (2011) \\
\hline$\hat{T}$ & temperature & 293 & $\mathrm{~K}$ & standard \\
\hline$\hat{R}_{g}$ & universal gas constant & 8.314 & $\mathrm{~Pa} \mathrm{~m}^{3} \mathrm{~mol}^{-1} \mathrm{~K}^{-1}$ & standard \\
\hline$\hat{z}$ & axial coordinate & variable & $\mathrm{m}$ & - \\
\hline$\hat{c}$ & concentration of sugar & variable & $\mathrm{mol} \mathrm{m}^{-3}$ & - \\
\hline$\hat{N}$ & concentration of sugar at $z=0$ & variable & $\mathrm{mol} \mathrm{m}^{-3}$ & Section 3 \\
\hline$\hat{F}$ & radial loading/unloading of sugar & variable & $\mathrm{mol} \mathrm{m}^{-2} \mathrm{~s}^{-1}$ & - \\
\hline$\hat{\eta}$ & passive loading parameter & variable & $\mathrm{m}^{-2} \mathrm{~s}^{-1}$ & - \\
\hline$\hat{\sigma}$ & active loading parameter & variable & $\mathrm{molm}^{-2} \mathrm{~s}^{-1}$ & - \\
\hline$\hat{f}$ & axial unloading of sugar at $z=z_{3}$ & variable & $\mathrm{mol} \mathrm{m}^{-2} \mathrm{~s}^{-1}$ & - \\
\hline$\hat{q}_{z}$ & axial flux & variable & $\mathrm{m}^{3} \mathrm{~s}^{-1}$ & - \\
\hline$\hat{q}_{r}$ & radial flux & variable & $\mathrm{m} \mathrm{s}^{-1}$ & - \\
\hline$\hat{p}$ & internal phloem pressure & variable & $\mathrm{Pa}$ & - \\
\hline$\hat{\Psi}_{e x t}$ & external water potential & 0 & $\mathrm{~Pa}$ & Section 2 \\
\hline$\hat{\Psi}$ & internal water potential & variable & $\mathrm{Pa}$ & - \\
\hline$\tilde{\Psi}_{\pi}$ & osmotic potential & variable & $\mathrm{Pa}$ & - \\
\hline
\end{tabular}

Table 1: Summary of dimensional symbols and their values.

as a reference set $\hat{\Psi}_{\text {ext }}=0$. We rewrite $\hat{\Psi}^{i}$ as a sum of the pressure, $\hat{p}^{i}$, and osmotic potential, $\hat{\Psi}_{\pi}(\mathrm{Pa})$. For simplicity we choose the Van't Hoff approximation (Kramer \& Boyer, 1995) of linear dependence on concentration, $\hat{\Psi}_{\pi}^{i}=-\hat{R}_{g} \hat{T} \hat{c}^{i}$, where $\hat{R}_{g}$ is the universal gas constant $\left(\mathrm{Pa} \mathrm{m}^{3} \mathrm{~mol}^{-1} \mathrm{~K}^{-1}\right)$ and $\hat{T}(K)$ is the temperature such that $\hat{\Psi}^{i}=\hat{p}^{i}-\hat{R}_{g} \hat{T} \hat{c}^{i}$. Note that nonlinear formulations of the osmotic potential are more accurate for high solute concentrations and have been used by Thompson \& Holbrook (2003a). By conservation of mass, the gradient of axial flow along the vessel balances the radial flow into the vessel such that

$$
-\hat{k}_{z} \frac{d^{2} \hat{p}^{i}}{d \hat{z}^{2}}=-2 \pi \hat{a} \hat{L}_{p}\left(\hat{p}^{i}-\hat{R}_{g} \hat{T} \hat{c}^{i}\right)
$$

where $2 \pi \hat{a} \hat{L}_{p}$ is the radial conductivity $\left(\mathrm{m}^{2} \mathrm{~Pa}^{-1} \mathrm{~s}^{-1}\right)$ of the phloem boundary. We follow the approach of Jensen et al. (2011, 2012) and assume that both the phloem tips (in the leaf and in the roots) are impermeable to water and hence prescribe zero flux of water:

$$
\hat{q}_{z}^{L}=0 \quad \text { at } \hat{z}=0, \quad \text { and } \quad \hat{q}_{z}^{R}=0 \quad \text { at } \hat{z}=\hat{z}_{3} .
$$


Note that the baseline value of pressure is set by the osmotic potential, $\hat{R}_{g} \hat{T} \hat{c}^{i}$. In addition, we prescribe continuity of the pressure $\hat{p}^{i}$, and the flux, $\hat{q}_{z}^{i}$, at the zone boundaries, $\hat{z}_{1}$ and $\hat{z}_{2}$.

\subsection{Sugar transport in the phloem}

Sugar is loaded into the phloem in the leaf region after production by photosynthesis and then, after transport, unloaded in the stem and root regions. Following the approaches of Thompson \& Holbrook (2003a), and Jensen et al. (2011, 2012), we assume that the loading and unloading of sugar into and out of the phloem vessels, which we represent by $\hat{F}^{i}\left(\mathrm{~mol} \mathrm{~m}^{-2} \mathrm{~s}^{-1}\right)$, is balanced by the flux of sugar in the axial direction. Similar to Payvandi et al. (2014) we also allow diffusion to contribute to the axial flux and allow unloading to also take place in the stem region. Leakage of solutes has been observed along the whole length of the phloem (Minchin \& Thorpe, 1987, 1996; Van Bel, 2003), and sugar stem reserves are of particular importance to crop grain filling (Rawson \& Evans, 1971). Therefore, conservation of $\hat{c}$ is given by

$$
\frac{d}{d \hat{z}}\left(\hat{c}^{i} \hat{q}_{z}^{i}-\pi \hat{a}^{2} \hat{D} \frac{d \hat{c}^{i}}{d \hat{z}}\right)=2 \pi \hat{a} \hat{F}^{i}
$$

where $\hat{D}$ is the diffusion coefficient $\left(\mathrm{m}^{2} \mathrm{~s}^{-1}\right)$ of sugar in the phloem sap. We allow the loading and unloading of sugar in the phloem vessels to be either active or passive. Active loading/unloading refers to input/output of sugar in the phloem independent of the sugar status within the phloem, representing the action of a pump. Consequently, active unloading can be unphysical for low $\hat{c}^{i}$. Conversely passive loading/unloading is dependent on the sugar status, and represents the transport of sugar down the sugar concentration gradient. We let

$$
\hat{F}^{i}=\hat{\eta}^{i} \hat{c}^{i}+\hat{\sigma}^{i}
$$

where, if $\hat{\sigma}^{i}\left(\mathrm{~m}^{-2} \mathrm{~s}^{-1}\right)$ is zero, the loading/unloading is passive. Conversely, if $\hat{\eta}^{i}\left(\mathrm{~mol} \mathrm{~m}^{-2} \mathrm{~s}^{-1}\right)$ is zero, the loading/unloading is active. In each zone, we allow the loading/unloading to be either completely active or passive. For both wheat and black locust, it is thought that the loading in the leaf zone is active, i.e sugar is pumped in (Jensen et al., 2013). Therefore throughout the paper we consider active loading in the leaf zone, and either active or passive unloading in the stem and root zones. Note that active loading is similar to the implicit loading of Jensen et al. (2012), whilst passive loading is similar to their explicit loading case.

Payvandi et al. (2014) showed diffusion to be important in the xylem vessels, and we expect that it may also be of importance in the phloem vessels especially near the ends of the phloem vessels when the fluid velocity falls 
to zero. The inclusion of diffusion also allows the modelling of an axial flux of sugar, $\hat{f}\left(\mathrm{~mol} \mathrm{~m}^{-2} \mathrm{~s}^{-1}\right)$, at the vessel termini in the roots $\left(\hat{z}=\hat{z}_{3}\right)$, which we include for generality. It is possible that axial effluxes of sugar occur due to the nutrient requirement of the meristemic tissue at the growing root tip (Bingham \& Stevenson, 1992). In addition, sugar exudates at the root tip (presumably from the phloem) can be important for promoting bacterial interactions in the soil (Dilkes et al., 2004). For the remaining boundary condition on $\hat{c}$, we prescribe the concentration of sugar to be $\hat{N}\left(\mathrm{~mol} \mathrm{~m}^{-3}\right)$ at the terminus in the leaf. The boundary conditions therefore are

$$
\hat{c}^{L}=\hat{N} \text { at } z=0, \quad \hat{c}^{R} \hat{q}_{z}^{R}-\pi \hat{v}_{j}^{R} \hat{a}_{j}^{R 2} \hat{D} \frac{\hat{d} c^{R}}{d \hat{z}}=\pi \hat{a}_{j}^{i 2} \hat{f} \text { at } \hat{z}=\hat{z}_{3} .
$$

In addition, at the zone boundaries, $\hat{z}_{1}$ and $\hat{z}_{2}$, we prescribe continuity and flux continuity of $\hat{c}^{i}$. In Section 3 we choose $\hat{N} \neq 0$ due to the loading of sugar at $\hat{z}=0$ after being produced by photosynthesis.

\section{Nondimensionalisation and parameter values}

We nondimensionalise the equations by using the following scales $\hat{z}=\hat{z}_{3} z$, $\hat{p}=\hat{P} p, \hat{c}=\hat{C} c$, where $\hat{P}$ is a typical internal pressure $(\mathrm{Pa})$ and $\hat{C}$ is a typical concentration of sugar $\left(\mathrm{mol} \mathrm{m}^{-3}\right)$. Note that only dimensional values are denoted with a hat $\left(^{\wedge}\right)$ symbol. Similarly to Phillips \& Dungan (1993) and Thompson \& Holbrook (2003b), we calculate $\hat{P}$ using the viscous pressure scale such that $\hat{P}=\hat{z}_{3} \hat{\mu} \hat{U} / \hat{a}^{2}$, where $\hat{U}$ is a typical sap velocity $\left(\mathrm{m} \mathrm{s}^{-1}\right)$. The nondimensional equations for water, and sugar transport are

$$
\begin{gathered}
\frac{d^{2} p^{i}}{d z^{2}}=M\left(p^{i}-H c^{i}\right), \\
\frac{d}{d z}\left(c^{i}\left(\frac{d p^{i}}{d z}\right)+\frac{1}{P e^{i}} \frac{d c^{i}}{d z}\right)=-F^{i},
\end{gathered}
$$

where the nondimensional parameters are defined in Table 2. $M$ is the Münch number (Jensen et al., 2009) defined as the ratio of radial conductivity of water to axial conductivity. $H$ is the osmotic pull of the sugars defined as the ratio of osmotic strength of the solution to the pressure scale (Phillips \& Dungan, 1993; Thompson \& Holbrook, 2003b). The Péclet number, Pe, is the ratio of convection to diffusion, and $F^{i}=\eta^{i} c^{i}+\sigma^{i}$ is the nondimensional loading/unloading parameter defined by a passive loading parameter, $\eta^{i}$, and an active loading parameter, $\sigma^{i}$.

The nondimensional boundary conditions are

$$
\begin{gathered}
\frac{d p^{L}}{d z}=0 \text { at } z=0, \quad \frac{d p^{R}}{d z}=0 \text { at } z=1, \quad c^{L}=\phi \text { at } z=0, \\
c^{R}\left(\frac{d p^{R}}{d z}\right)+\frac{1}{P e} \frac{d c^{R}}{d z}=-f \text { at } z=1,
\end{gathered}
$$


where $\phi$ is the nondimensional concentration at $z=0$ of sugar in the phloem, and $f$ is the axial unloading parameter of sugar through the phloem tip in the root at $z=1$ (see Table 2 for definitions). We also specify continuity and flux continuity of $p^{i}$ and $c^{i}$ at the zone boundaries, $z_{1}$ and $z_{2}$.

\begin{tabular}{|c|c|c|c|}
\hline Symbol & Description & Wheat Value & Black Locust Value \\
\hline \hline$z_{1}=\frac{\hat{z}_{1}}{\hat{z}_{3}}$ & nondimensional leaf-stem boundary & 0.3 & 0.2 \\
\hline$z_{2}=\frac{z_{2}}{\hat{z}_{3}}$ & nondimensional stem-root boundary & 0.4 & 0.8 \\
\hline$M=\frac{16 \hat{\mu} \hat{L}_{p} \hat{z}_{3}^{2}}{\hat{a}^{3}}$ & ratio of radial conductivity to axial conductivity & 12 & $1 \times 10^{4}$ \\
\hline$H=\frac{\hat{R}_{g} \hat{T} \hat{C}}{\hat{P}}$ & ratio of osmotic forces to viscous losses & 95 & $2.5 \times 10^{6}$ \\
\hline$P e=\frac{\hat{k}_{z} \hat{P}}{\pi \hat{a}^{2} \hat{D}}$ & ratio of convective to diffusive transport & $4 \times 10^{4}$ & $*$ \\
\hline$F^{L}=\frac{2 \pi \hat{a} \hat{z}_{3}^{2} \hat{F}^{L}}{\hat{k}_{z} \hat{P} \hat{C}^{2}}$ & ratio of leaf radial loading to axial transport & $*$ & $*$ \\
\hline$F^{S}=\frac{2 \pi \hat{a} \hat{z}_{3}^{2} \hat{F}^{S}}{\hat{k}_{z} \hat{P} \hat{C}^{2}}$ & ratio of leaf radial loading to axial transport & $*$ & $*$ \\
\hline$F^{R}=\frac{2 \pi \hat{a} \hat{z}_{3}^{2} \hat{F}^{R}}{\hat{k}_{z} \hat{P} \hat{C}}$ & ratio of leaf radial loading to axial transport & $*$ & $*$ \\
\hline$f=\frac{\pi \hat{a}^{2} \hat{z}_{3} \hat{f}}{\hat{k}_{z} \hat{P} \hat{C}}$ & ratio of axial loading to axial transport & $*$ & $*$ \\
\hline$\phi=\frac{\hat{N}}{\hat{C}}$ & ratio of sugar concentration scales at $z=0$ & $*$ & $*$ \\
\hline
\end{tabular}

Table 2: Summary of nondimensional symbols and typical values used, based on dimensional values given in Table 1. The starred symbol $\left({ }^{*}\right)$ indicates that the value will be varied throughout the paper (see text in Section 3 for discussion).

To calculate the values of the nondimensional parameters in Table 2, we use values for wheat and black locust given in Table 1. Phloem sap velocities are generally in the range 0.5 to $1 \mathrm{~m} \mathrm{hr}^{-1}$ (Thompson \& Holbrook, 2003a), and we use an average value of $U=2 \times 10^{-4} \mathrm{~m} \mathrm{~s}^{-1}$. We use wheat dimensions measured from Kutschera et al. (2009) and black locust dimensions from Jensen et al. (2011), where we use $\hat{z}_{2}=40 \mathrm{~m}$ and estimate $\hat{z}_{1}=10 \mathrm{~m}$, and $\hat{z}_{3}=50 \mathrm{~m}$ from typical leaf-stem-root proportions used in Jensen et al. (2011). Due to the difference in height between wheat and black locust we obtain a very large range in $M\left(M=12\right.$ for wheat and $M=10^{4}$ for black locust). Most plants fall within this range (Jensen et al., 2011) and when $M$ is large, the plants are in a state of osmotic equilibrium with their surroundings (Thompson \& Holbrook, 2003a). The Péclet number is $P e=4 \times 10^{4}$ for wheat and $P e=2.5 \times 10^{6}$ for black locust. This indicates that diffusion is small compared to convection in the bulk flow, for both plants, in the absence of boundaries.

In general, the dimensional loading function of sugar, $\hat{F}$, is unknown in the literature. We follow the approach of Jensen et al. (2012); Payvandi et al. (2014) to investigate the effect of varying the values of the nondimensional radial and axial loading parameters $F$, and $f$. To represent the loading of sugar in the leaf due to photosynthesis, and unloading in the stem and root zones for storage and growth, we prescribe that $F^{L}$ is positive whilst $F^{S}$ and $F^{R}$ are both negative. In addition, we let $f$ be positive to represent 
axial efflux of sugar out of the phloem vessels to be used for root growth.

To solve across the parameter regimes applicable for wheat and black locust we solve for the phloem transport both analytically and numerically. The numerical computations are conducted in Matlab using the pdepe solver where equations (8) and (9) are solved subject to the boundary conditions (10). In Section 4 we will discuss the analytical method and solution, and in Section 5 we will compare the analytical and numerical solutions.

\section{Analytical solution}

Following the approach of Jensen et al. (2012) we first differentiate equation (8) and rewrite $p$ in terms of flux, $w^{i}=\frac{d p^{i}}{d z}$, in (8) and (9) to give

$$
\begin{gathered}
\frac{d^{2} w^{i}}{d z^{2}}=M\left(w^{i}-H \frac{d c^{i}}{d z}\right), \\
\frac{d c^{i}}{d z} w^{i}+c^{i} \frac{d w^{i}}{d z}+\frac{1}{P e} \frac{d^{2} c^{i}}{d z^{2}}=-F^{i} .
\end{gathered}
$$

The boundary conditions (10) written in terms of $w$ are

$$
\begin{gathered}
w^{L}=0, \quad c^{L}=\phi, \quad \text { at } \quad z=0, \\
w^{R}=0, \quad c^{R} w^{R}+\frac{1}{P e} \frac{d c^{R}}{d z}=-f \quad \text { at } \quad z=1 .
\end{gathered}
$$

The conditions of continuity become continuity of $c, w$, and flux of $c$ at the zone boundaries $z_{1}$ and $z_{2}$. Note that as $p$ and $c$ are continuous at $z=z_{1}$ and $z=z_{2}$ then, using equation (8), $\frac{d^{2} p}{d z^{2}}=\frac{d w}{d z}$ is also continuous.

To analytically solve equations (11) and (12) we use the method of matched asymptotic expansions and consider the limit of high $M$ and high $P e$ which characterises most plants. For high $M$ and $P e$, the water and nutrient transport equations become singular since the highest derivatives are multiplied by $\frac{1}{M}$ or $\frac{1}{P e}$ respectively. In order to regularise equations (11) and (12), we seek boundary layers in the regions where the derivatives become large; at the ends of the phloem or at the zone boundaries. We then solve in the boundary layer regions and the central region separately, and finally match the solutions together. For an introduction to boundary layer methods and matched asymptotics, see Hinch (1991). Note that previous authors (Thompson \& Holbrook, 2003a; Jensen et al., 2012) neglect diffusion entirely. This is equivalent to considering $P e=\infty$ and, hence, neglecting boundary layers in the solution for $c$.

To solve in the leaf, stem and root zones, we split each zone into one central bulk region, one left-hand-side boundary (LHS), and one right-handside boundary (RHS), which results in 9 regions in total given in Figure 1. In the bulk regions convection dominates and $w^{i}=H \frac{d c^{i}}{d z}\left(\right.$ and $p^{i}=H c^{i}$ ), corresponding to osmotic equilibrium, whereas in the boundary layers, diffusion 


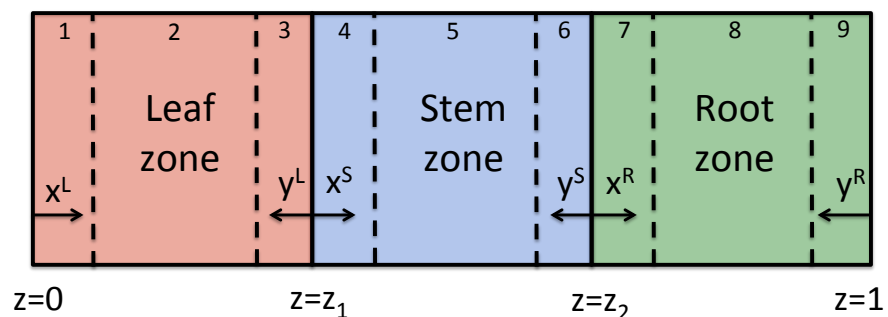

Figure 1: Nondimensional model of a phloem vessel. $z=0$ is the top of the phloem in the leaf, $z_{1}$ is the leaf-stem boundary, $z_{2}$ is the stem-root boundary, and $z_{3}$ is the phloem terminus in the root. $x^{i}$ are local boundary layer coordinates in the LHS boundaries of each zone, and $y^{i}$ are local boundary layer coordinates in the RHS boundaries of each zone.

of $c$ and $p$ are also important. We therefore seek boundary layer solutions near $z=0$ and $z=1$ to satisfy the boundary conditions at the phloem ends, and also at the zone boundaries $z_{1}$ and $z_{2}$ to satisfy the continuity boundary conditions.

We initially consider the leaf, stem and root zones separately, but in all zones and regions we apply the following scalings

$$
\begin{gathered}
\epsilon=1 / M, \quad 1 / P e=\epsilon^{\chi} / \bar{P} e, \quad w^{i}=\epsilon^{\delta} \bar{w}^{i}, \quad H=\epsilon^{\beta} \bar{H}, \\
F^{i}=\epsilon^{\gamma} \bar{F}^{i}, \quad \eta=\epsilon^{\gamma} \bar{\eta}, \quad \sigma=\epsilon^{\gamma} \bar{\sigma} \quad f=\epsilon^{\gamma} \bar{f},
\end{gathered}
$$

such that $\bar{P} e, \bar{w}^{i}, \bar{F}^{i}, \bar{\eta}, \bar{\sigma}, \bar{f}$, and $\bar{H}$ are $O(1)$ and the values of $\delta, \gamma, \beta$, and $\chi$, are as yet undetermined. The structure of the equations is the same in each zone, therefore we can write the equations in a general form. In the leaf, stem, and root zones, the left-hand-side boundaries are $z=0, z=z_{1}$, and $z=z_{2}$ respectively, and we rewrite $z$ in terms of a boundary layer variable $x^{i}$, such that

$$
z=\epsilon^{\alpha} x^{L}, \quad z=z_{1}+\epsilon^{\alpha} x^{S}, \quad z=z_{2}+\epsilon^{\alpha} x^{R},
$$

in the leaf, stem, and root zones respectively. Similarly, the right-hand-side boundaries respectively are $z=z_{1}, z=z_{2}$, and $z=1$, and we rewrite $z$ in terms of a boundary layer variable $y^{i}$, such that

$$
z=z_{1}-\epsilon^{\alpha} y^{L}, \quad z=z_{2}-\epsilon^{\alpha} y^{S}, \quad z=1-\epsilon^{\alpha} y^{R} .
$$

The scaled equations in the bulk region, LHS boundary region, and RHS boundary region for all zones are given in Appendix A, together with the boundary conditions at $z=0$ and $z=1$, and continuity conditions at $z=z_{1}$ and $z=z_{2}$.

We choose values for the scales by balancing terms in the equations against each other. In the fluid transport equations (equations (A.3) and 
(A.5)), we choose $\alpha=\frac{1}{2}$ to balance the viscous flow terms $\left(\frac{d^{2} \bar{w}^{i}}{d x^{i 2}}\right.$ and $\left.\frac{d^{2} \bar{w}^{i}}{d y^{i 2}}\right)$ with the convective term, $\bar{w}^{i}$. Similarly, we choose $\beta=\chi$ to balance the viscous flow in equations (A.3) and (A.5) with convection of $c\left(\frac{d c^{i}}{d x^{i}} \bar{w}^{i}, \frac{d c^{i}}{d y^{i}} \bar{w}^{i}\right.$, $\left.c^{i} \frac{d \bar{w}^{i}}{d x^{i}}, c^{i} \frac{d \bar{w}^{i}}{d y^{i}}\right)$ in equations (A.4) and (A.6). Since convection of $c$ falls to zero in the boundary layers, it is necessary that diffusion of $c\left(\frac{d^{2} c^{i}}{d x^{i, 2}}\right.$ and $\left.\frac{d^{2} c^{i}}{d y^{i, 2}}\right)$ dominate in equations (A.4) and (A.6), and this is achieved by choosing $\delta=1+\chi-\alpha$. Finally, in order to make analytical progress, we choose $\gamma$ such that the loading and unloading are small. In summary, we obtain

$$
\alpha=\frac{1}{2}, \quad \beta=\chi, \quad \delta=1+\chi-\alpha, \quad \gamma=\frac{3}{2}-2 \alpha+\chi .
$$

Considering black locust as an example, where $M=10^{4}$ and $P e \approx 10^{6}$ then $\chi=1.5$ which gives $\beta=1.5, \delta=2, \gamma=2$. Note that for this value of $\beta$, the scaling of $H$ becomes inconsistent with the biological estimations of $H$ given in Table 2. However, we will show in Section 5 in Figures 3 and 4, that despite this scaling, the analytical solution is in good agreement with the numerical solution for biological values of $H$. The fluid transport and concentration transport equations scaled for black locust are

$$
\begin{gathered}
\epsilon^{\frac{3}{2}} \frac{d^{2} \bar{w}^{i}}{d z^{2}}=\epsilon^{\frac{1}{2}} \bar{w}^{i}-\bar{H} \frac{d c^{i}}{d z}, \\
\epsilon^{\frac{1}{2}} \frac{d c^{i}}{d z} \bar{w}^{i}+\epsilon^{\frac{1}{2}} c^{i} \frac{d \bar{w}^{i}}{d z}+\frac{1}{\bar{P} e} \frac{d^{2} c^{i}}{d z^{2}}=-\epsilon^{\frac{1}{2}} \bar{F}^{i}
\end{gathered}
$$

in the bulk region.

$$
\begin{gathered}
\epsilon \frac{d^{2} \bar{w}^{i}}{d x^{i 2}}=\epsilon \bar{w}^{i}-\bar{H} \frac{d c^{i}}{d x^{i}}, \\
\epsilon \frac{d c^{i}}{d x^{i}} \bar{w}^{i}+\epsilon c^{i} \frac{d \bar{w}^{i}}{d x^{i}}+\frac{1}{\bar{P} e} \frac{d^{2} c^{i}}{d x^{i, 2}}=-\epsilon^{\frac{3}{2}} \bar{F}^{i},
\end{gathered}
$$

in the LHS boundary Region, where $z=\epsilon^{\frac{1}{2}} x^{L}$ in the leaf, $z=z_{1}+\epsilon^{\frac{1}{2}} x^{S}$ in the stem, and $z=z_{2}+\epsilon^{\frac{1}{2}} x^{R}$ in the root. Finally,

$$
\begin{gathered}
\epsilon \frac{d^{2} \bar{w}^{i}}{d y^{i 2}}=\epsilon \bar{w}^{i}+\bar{H} \frac{d c^{i}}{d y^{i}} \\
-\epsilon \frac{d c^{i}}{d y^{i}} \bar{w}^{i}-\epsilon c^{i} \frac{d \bar{w}^{i}}{d y^{i}}+\frac{1}{\bar{P} e} \frac{d^{2} c^{i}}{d y^{i 2}}=-\epsilon^{\frac{3}{2}} \bar{F}^{i} .
\end{gathered}
$$

in the RHS boundary Region, where $z=z_{1}-\epsilon^{\frac{1}{2}} y$ in the leaf, $z=z_{2}-\epsilon^{\frac{1}{2}} y$ in the stem, and $z=1-\epsilon^{\frac{1}{2}} y$ in the root. The scaled boundary conditions become

$$
\bar{w}^{L}=0, \quad c^{L}=\phi, \quad \text { at } \quad x^{L}=0,
$$




$$
\bar{w}^{R}=0, \quad \frac{d c}{d y^{R}}=\epsilon \bar{P} e \bar{f} \quad \text { at } \quad y^{R}=0 .
$$

The scaled continuity conditions at the leaf-stem boundary $z=z_{1}$ are

$$
\begin{gathered}
c^{L}=c^{S}, \quad \bar{w}^{L}=\bar{w}^{S}, \\
\epsilon^{\frac{1}{2}} c^{L} \bar{w}^{L}+\frac{1}{\bar{P} e} \frac{d c^{L}}{d z}=\epsilon^{\frac{1}{2}} c^{S} \bar{w}^{S}+\frac{1}{\bar{P} e} \frac{d c^{S}}{d z}, \quad \frac{d \bar{w}^{L}}{d z}=\frac{d \bar{w}^{S}}{d z},
\end{gathered}
$$

an the scaled continuity conditions at the stem-root boundary $z=z_{2}$ are

$$
\begin{gathered}
c^{S}=c^{R}, \quad \bar{w}^{S}=\bar{w}^{R}, \\
\epsilon^{\frac{1}{2}} c^{S} \bar{w}^{S}+\frac{1}{\bar{P} e} \frac{d c^{S}}{d z}=\epsilon^{\frac{1}{2}} c^{R} \bar{w}^{R}+\frac{1}{\bar{P} e} \frac{d c^{R}}{d z}, \quad \frac{d \bar{w}^{S}}{d z}=\frac{d \bar{w}^{R}}{d z} .
\end{gathered}
$$

In the bulk region (equations 19 and 20) the osmotic potential and the diffusion of $c$ dominate. The viscous flow terms are much smaller indicating that, in the bulk region, viscous effects are small compared to osmotic effects.

In the LHS and RHS boundary regions, the terms that dominate are the osmotic potential terms $\left(\bar{H} \frac{d c^{i}}{d x^{i}}\right.$ and $\left.\bar{H} \frac{d c^{i}}{d y^{2}}\right)$ in equations (21) and (23), and the diffusive transport of $c\left(\frac{1}{\bar{P} e} \frac{d^{2} c^{i}}{d x^{i, 2}}\right.$ and $\left.\frac{1}{\bar{P} e} \frac{d^{2} c^{i}}{d y^{i, 2}}\right)$ in equations (22) and (24). In addition, the viscous flow terms $\left(\frac{d^{2} \bar{w}^{i}}{d x^{i 2}}\right.$ and $\left.\frac{d^{2} \bar{w}^{i}}{d y^{i 2}}\right)$ are now at the same order of $\epsilon$ as the radial flow (in the fluid transport equations) and the convection of $c$ (in the concentration transport equations).

We expand $c^{i}, \bar{w}^{i}, \bar{F}$ in increasing powers of $\epsilon^{\frac{1}{2}}$ such that

$$
\begin{gathered}
c^{i}=c_{0}^{i}+\epsilon^{\frac{1}{2}} c_{1}^{i}+\epsilon c_{2}^{i}+\epsilon^{\frac{3}{2}} c_{3}^{i}+O\left(\epsilon^{2}\right), \\
\bar{w}^{i}=\bar{w}_{0}^{i}+\epsilon^{\frac{1}{2}} \bar{w}_{1}^{i}+\epsilon \bar{w}_{2}^{i}+\epsilon^{\frac{3}{2}} \bar{w}_{3}^{i}+O\left(\epsilon^{2}\right), \\
\bar{F}^{i}=\bar{F}_{0}^{i}+\epsilon^{\frac{1}{2}} \bar{F}_{1}^{i}+O(\epsilon), \\
\bar{F}_{0}^{i}=\bar{\eta}^{i} c_{0}^{i}+\bar{\sigma}^{i}, \quad \bar{F}_{2}^{i}=\bar{\eta}^{i} c_{1}^{i} .
\end{gathered}
$$

We solve the fluid transport and concentration transport equations in all regions subject to the boundary and continuity conditions at increasing orders of $\epsilon$. In general the solution process involves solving in each region first, applying the boundary conditions, then matching the solutions within each zone to obtain a composite solution. Finally, we apply continuity of $c^{i}, w^{i}$ and flux of $c^{i}$ and $w^{i}$ at the zone boundaries $z_{1}$ and $z_{2}$, to determine the unknown constants and the solution for all zones. The solution procedure for $O\left(\epsilon^{0}\right)$ and $O\left(\epsilon^{\frac{1}{2}}\right)$ is given in Appendix $\mathrm{B}$, and the solutions for $c_{0}^{i}, c_{1}^{i}$, $c_{2}^{i}, \bar{w}_{0}^{i}, \bar{w}_{1}^{i}$ are given in Appendix C. To calculate the pressure, we apply the scalings from equation (15) to equation (8) such that

$$
p^{i}=\epsilon^{\beta} \bar{H} c^{i}+\epsilon^{1+\delta} \frac{d \bar{w}^{i}}{d z} .
$$


Expanding $c^{i}$ and $\bar{w}^{i}$ gives the solution for $p^{i}$ up to $O(\epsilon)$

$$
p^{i}=\epsilon^{\beta}\left(\bar{H} c_{0}^{i}+\epsilon^{\frac{1}{2}} \bar{H} c_{1}^{i}+\epsilon \bar{H} c_{2}^{i}+\epsilon^{\frac{3}{2}}\left(\bar{H} c_{3}+\frac{d \bar{w}_{0}}{d z}\right)+O\left(\epsilon^{2}\right)\right) .
$$

Note that $p$ is automatically continuous across the zone boundaries since equation (35) is continuous across the zone boundaries.

The largest term in $c^{i}$ is given by $c_{0}^{i}$ (equation (C.1)) and arises due to the domination of the osmotic potential $\left(\bar{H}^{d c_{0}^{i}} \frac{1}{d z}\right)$ in all three zones. Since the osmotic potential is the only term at $O\left(\epsilon^{0}\right)$ in the fluid transport equation (equation (B.3)), $c_{0}^{i}$ is constant and depends only on the boundary condition on $c^{L}$ applied at $z=0$. The remainder of terms for $c^{i}$ represent deviations away from this base value and arise from interactions of the osmotic potential with other processes. For example, at $O\left(\epsilon^{\frac{1}{2}}\right)$, the osmotic potential again dominates in the boundary layer regions (equation (B.4)), whereas in the bulk regions, the osmotic potential of $c_{1}^{i}$ is balanced by the the axial velocity, $\bar{w}_{0}^{i}$, and the diffusion of $c_{1}^{i}$ is driven by the convection of $c_{0}^{i}$ due to $\bar{w}_{0}^{i}$ and the loading/unloading due to $\bar{F}_{0}$ (equation (B.10)). At $O(\epsilon)$, in the boundary layer regions, the viscous flow terms finally contribute to the fluid transport equations to drive the osmotic potential due to $c_{2}^{i}$ (equation (B.5)), and diffusion of $c_{2}^{i}$ is balanced by convection of $c_{0}^{i}$ due to $\bar{w}_{0}^{i}$ (equation (B.6)).

The first boundary layer terms, given by the exponentials, appear only in $c_{2}^{i}$ (equations (C.5) to (C.7)). However these terms drive the bulk (nonexponential) terms in $c_{1}^{i}$ (equations (C.2) to (C.4)). The implication of this, is, that diffusion influences the bulk solution as well the boundary layer solutions. The exponential terms in $c_{2}^{i}$ are due to the boundary conditions at $z=0$ and $z=1$, whilst the exponential terms due to the zone boundaries do not appear until $c_{3}^{i}$ (equations (C.8) to (C.10)). In contrast to $c^{i}$, the leading order terms of $w^{i}$ are dependent on $\epsilon$ and $P e$ since $w=\epsilon^{\delta} \bar{w} . \bar{w}_{0}^{i}$ arises due to osmotic equilibrium in the bulk regions, and the balance of viscous flow with radial flow in the boundary layer regions. In addition, the boundary layer terms due to $z=0$ and $z=1$ appear at leading order in $\bar{w}_{0}^{i}$ (equations (C.11) to (C.13)), whereas the zone boundary terms appear at first order in $\bar{w}_{1}^{i}$ (equations (C.14) to (C.16)).

The structure of the analytical solution is conserved for all $M$ and $P e$ as long as $H$ and $F^{i}$ are scaled according to equation (15). The analytical solution is valid for all $P e$, and as $P e$ increases (holding $M, \bar{H}$, and $\bar{F}^{i}$ constant) the main effect is that the magnitude of $w^{i}$ decreases as $\chi$ increases.

\section{Results}

\subsection{Comparison of analytical to numerical solution}

We consider $M=10^{4}$ and $P e=10^{6}$, which are similar values to that for black locust, $\chi=1.5, H=O\left(10^{-6}\right)$ and $F=O\left(10^{-8}\right)$. The load- 
ing/unloading is represented by $\sigma=\left(\sigma^{L}, \sigma^{S}, \sigma^{R}\right)$ and $\eta=\left(\eta^{L}, \eta^{S}, \eta^{R}\right)$, where the superscript denotes the zone. For example $\sigma^{L}$ represents the active loading in the leaf zone, $\sigma^{S}$ represents the active loading in the stem zone, and $\sigma^{R}$ represents the active loading in the root zone.

A comparison of the analytical solution to the numerical solution for these parameter values is shown in Figure 2. The comparison shows that the analytic approximation accurately captures the behaviour of $c, w$, and $p$, where the normalised L2-norm for $c$ is equal to $4.51 \times 10^{-7}$. In Figure

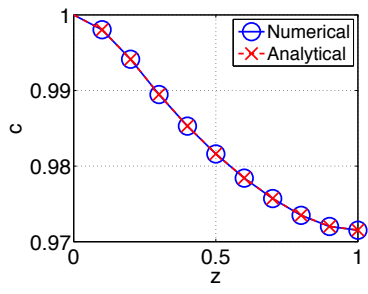

(a) concentration, $c$

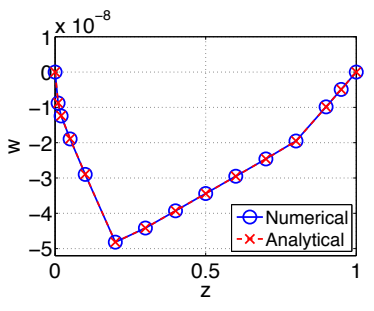

(b) velocity, $w$

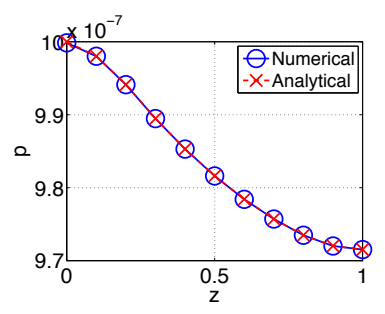

(c) pressure, $p$

Figure 2: Profiles of phloem concentration, $c$, velocity, $w$, pressure, $p$, and radial flow, $p-H c$, against axial distance, $z$, comparing the numerical solution (blue) to the analytical solution (red) for $M=10^{4}, P e=10^{6}$, $H=10^{-6}, z_{1}=0.2, z_{2}=0.8, f=0, \phi=1, \sigma=\left(4 \times 10^{-7}, 0,0\right)$, $\eta=\left(0,-1 \times 10^{-7},-2 \times 10^{-7}\right)$.

2(a) we see that the concentration of sugar, $c$, decreases with axial distance $z$; this reduction is due to the input of sugar into the phloem at the leaf zone ( $F^{L}$ is positive) and the output of sugar in the stem and root zones $\left(F^{S}\right.$ and $F^{R}$ are negative). The input and output of $c$ changes the osmotic potential within the phloem, causing inflow water at the leaf, and outflow in the stem and root zones. This causes pressure (Figure 2(c)) to be higher in the leaf zone than in the root zone, driving flow (Figure 2(b)) in that direction with the maximum velocity occurring at the leaf-stem boundary. We can see the development of a boundary layer near $z=0$ in $w$, but not in $c$ at $z=0$. This is as expected since the boundary layer terms appear at higher orders in the series in $c$ than for $w$.

We now consider the case where $H$ is not determined by the scaling in (15). Instead we use the values for black locust as given in Table 2 and plot, in Figure 3, the analytical and numerical solution for black locust parameter values, for the cases of active unloading $\left(\eta^{i}=0\right)$ and passive unloading $\left(\eta^{i} \neq 0\right)$. Note that the profile of $p$ has not been plotted since its behaviour is very similar to that of $c$. We consider three different magnitudes of loading and unloading parameters; $F_{A}=\eta c+\sigma, F_{B}=5 \times F_{A}$, and $F_{C}=10 \times F_{A}$ where $\eta=(0,0,0), \sigma=\left(4 \times 10^{-3},-1 \times 10^{-3},-2 \times 10^{-3}\right)$ for the active case, and $\eta=\left(0,-1 \times 10^{-3},-2 \times 10^{-3}\right), \sigma=\left(4 \times 10^{-3}, 0,0\right)$ for the passive case. We see that for $F=F_{A}$ and $F=F_{B}$, the analytical solution still agrees 

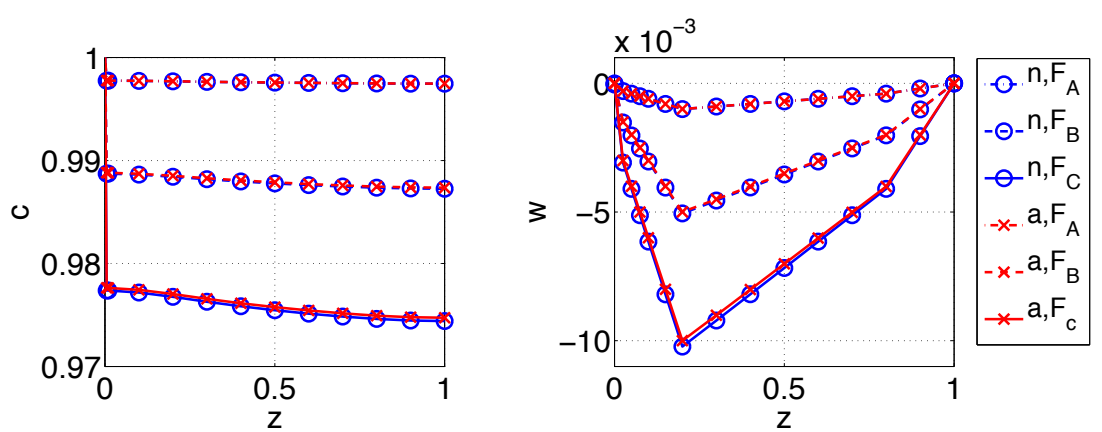

(a) Active unloading, $\eta=(0,0,0), \sigma=\left(4 \times 10^{-3},-1 \times 10^{-3},-2 \times 10^{-3}\right)$
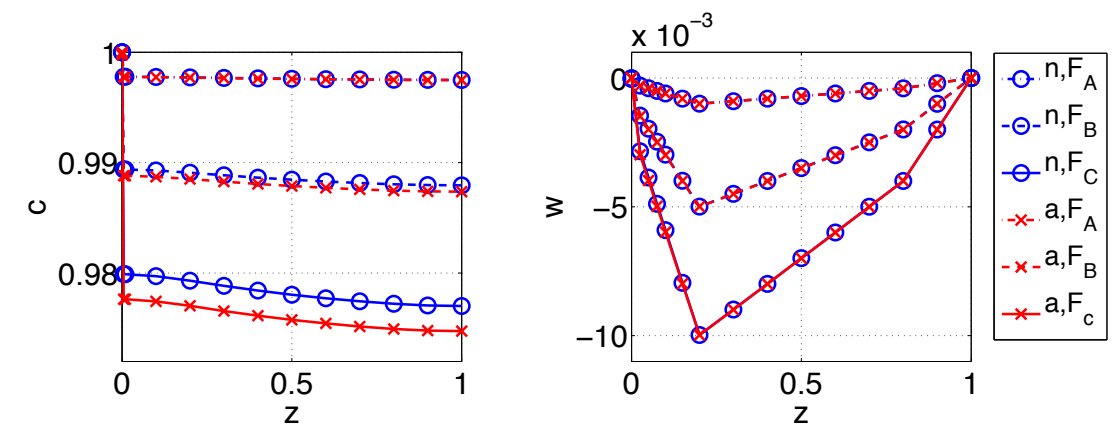

(b) Passive unloading, $\eta=\left(0,-1 \times 10^{-3},-2 \times 10^{-3}\right), \sigma=\left(4 \times 10^{-3}, 0,0\right)$

Figure 3: Profiles of phloem concentration, $c$, and velocity, $w$, against axial distance, $z$, comparing the numerical solution, $n$, to the analytical solution, a, for black locust parameter values where $M=10^{4}$, $P e=2.5 \times 10^{6}, H=2, z_{1}=0.2, z_{2}=0.8$, and $f=0, \phi=1$. The value of the loading/unloading parameter, $F$, is varied from $F_{A}$ where $F_{A}=\eta c+\sigma$, to $F_{B}=5 \times F_{A}$ and $F_{C}=10 \times F_{A}$. 
well with the numerical solution. Analytically, for active loading only, we see that

$$
\begin{array}{cc}
\epsilon^{0} c_{0}^{i} \gg \epsilon^{\frac{1}{2}} c_{1}^{i}, & \epsilon^{0} \bar{w}_{0}^{i} \gg \epsilon^{\frac{1}{2}} \bar{w}_{1}^{i}, \\
\Rightarrow \phi \gg \epsilon^{\frac{1}{2}}\left(\frac{\bar{P} e \bar{F}_{0}^{i}}{\kappa}\right), & 1 \gg \epsilon^{\frac{1}{2}}\left(\frac{\bar{H} \bar{F}_{0}^{i} \bar{P} e^{2}}{\kappa^{2}}\right),
\end{array}
$$

where $\kappa=\bar{H} \bar{P} e \phi+1$. If $\bar{P} e=\phi=1$ and $H=O(1)$, Equation (38) holds if $F_{0}^{i}=O\left(\epsilon^{\frac{1}{2}} H\right)$. We see in Figure 3 that the analytical solution compares well with the numerical solution when $F=O\left(\epsilon^{\frac{1}{2}}\right)\left(F=F_{A}\right)$ but starts to differ as $F$ increases. Increasing $F$ decreases the validity of the series, and for $F=F_{C}$ the analytical solution differs from the numerical solution. This difference is only small when the unloading is active, but becomes significant when the unloading is passive.

Considering the parameters specific to wheat, for which the value of $M$ is quite small $(M=12)$, in Figure 4 we see that for active unloading, the analytical solution provides a reasonable approximation to the magnitude of the numerical solution for $c$. Conversely, for passive unloading the analytical solution of $c$ is very different to the numerical solution. This is because the value of $M$ is too small for the asymptotic solution to be valid, and we can see that the analytical solution improves in accuracy as $M$ increases. Therefore, for small plants such as wheat the analytical solution might only be appropriate when the unloading is active. The value of $H$ is larger for wheat than for black locust, and hence for the analytical solution to be valid, the size of $F$ is larger.

For both wheat and black locust parameter values we can see that boundary layers are very sharp, particularly with regards to $c$ near $z=0$, and this boundary layer is responsible for the changing magnitude of $c$ near $z=0$. The analytical solution can be easily used to provide an estimate of the magnitude of $c$ near $z=0$. Neglecting the exponential terms in $c^{L}$, the analytical solution of $c^{L}$ at $z=0$ is

$$
\begin{gathered}
c_{0}^{L}=\phi \\
\epsilon^{\frac{1}{2}} c_{1}^{L}=0 \\
\epsilon c_{2}^{L}=\frac{1}{M^{0.5}} \frac{P e^{2} H \phi\left(z_{1}\left(F_{0}^{L}-F_{0}^{S}\right)+z_{2}\left(F_{0}^{S}-F_{0}^{R}\right)+F_{0}^{R}-f\right)}{(P e H \phi+1)^{\frac{3}{2}}} .
\end{gathered}
$$

We see that $\epsilon c_{2}^{L}$ is responsible for causing the deviation from $c_{0}^{L}=\phi$. The magnitude of $\epsilon c_{2}^{L}$ is approximately $O\left(M^{-0.5} H^{-0.5} \mathrm{Pe}^{0.5} \mathrm{~F}^{1}\right)$. Therefore, increasing either $M$ or $H$ reduces the magnitude of $c_{2}^{L}$ thereby reducing the deviation from $c_{0}^{L}=\phi$, whereas increasing $P e$ or $F$ increases the magnitude of $c_{2}^{L}$ increasing the deviation from $c_{0}^{L}$. 

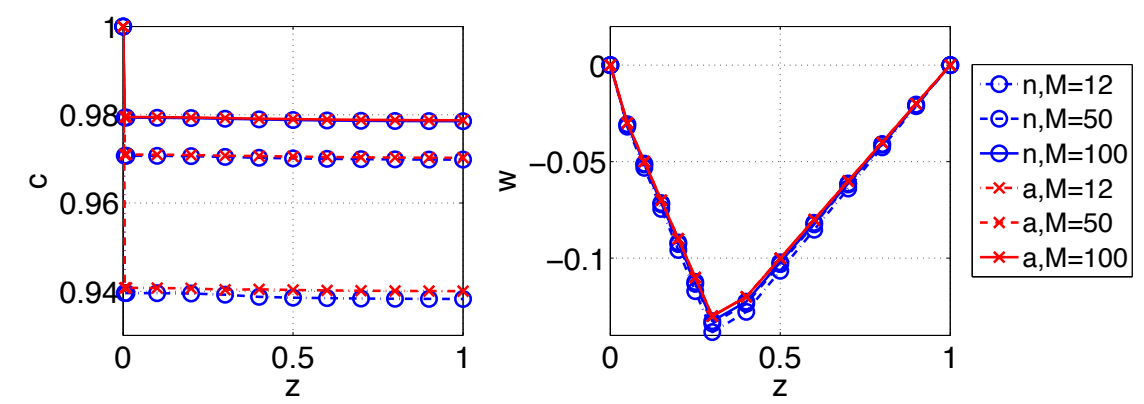

(a) Active unloading, $\eta=(0,0,0), \sigma=(0.4,-0.1,-0.2)$
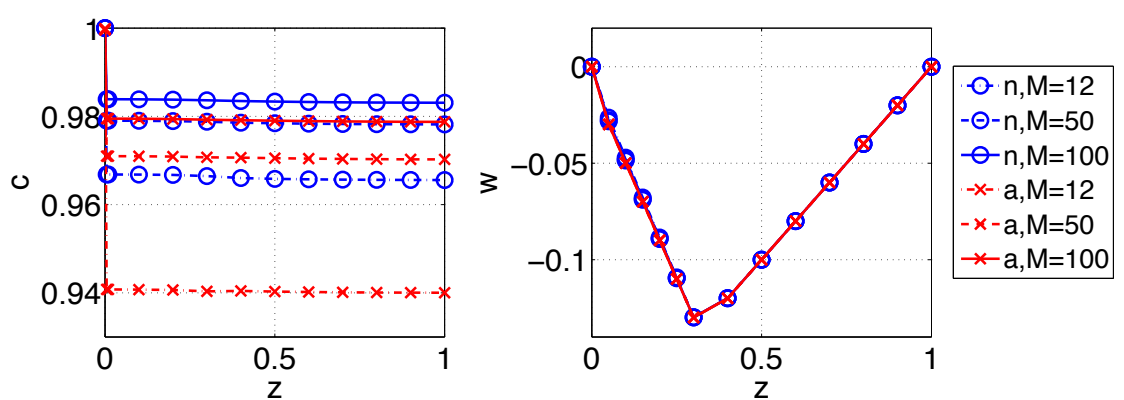

(b) Passive unloading, $\eta=(0,-0.1-0.2), \sigma=(0.4,0,0)$.

Figure 4: Profiles of phloem concentration, $c$, and velocity, $w$, against axial distance, $z$, comparing the numerical solution, $n$, to the analytical solution, a, for wheat parameter values where $P e=4 \times 10^{4}, H=95$, $z_{1}=0.3, z_{2}=0.4$, and $f=0, \phi=1$. The value of $M$ is varied to show the improvement of the analytical solution compared to the numerical solution for increasing $M$.

\subsection{Dependence of solution on $P e$}

In the previous section we saw that the analytical solution presented is valid only for high $M$, high $P e$ and small $F$. We now consider the numerical solution only, which is valid for all parameter regimes, to investigate the effect of varying the two main nondimensional parameters, $P e$ and $M$. We first consider the effect of $P e$ on the transport within the phloem to see if diffusion can be neglected. We will show that the validity of this assumption depends on the type and magnitude of loading.

Figure 5 shows the variation of $c$ and $w$ with increasing $P e$ for different values of $F$ comparing active to passive unloading, and we see that, generally, increasing $P e$ increases the deviation of $c$ from $c=\phi$, and increases the magnitude of $w$.

The majority of previous authors (Thompson \& Holbrook, 2003a; Jensen et al., 2012) modelling the phloem consider diffusion to be negligible and hence discard the diffusive terms, which corresponds to the limit $P e=\infty$. 

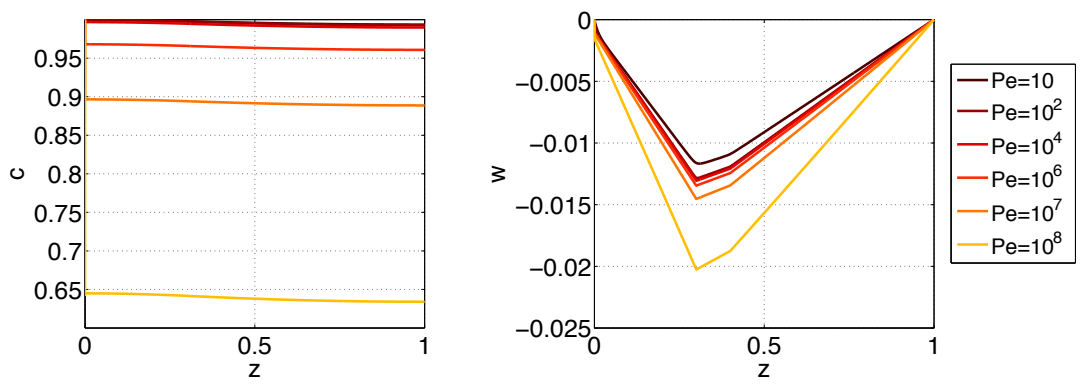

(a) Active unloading, $\eta=(0,0,0), \sigma=(0.04,-0.01,-0.02)$
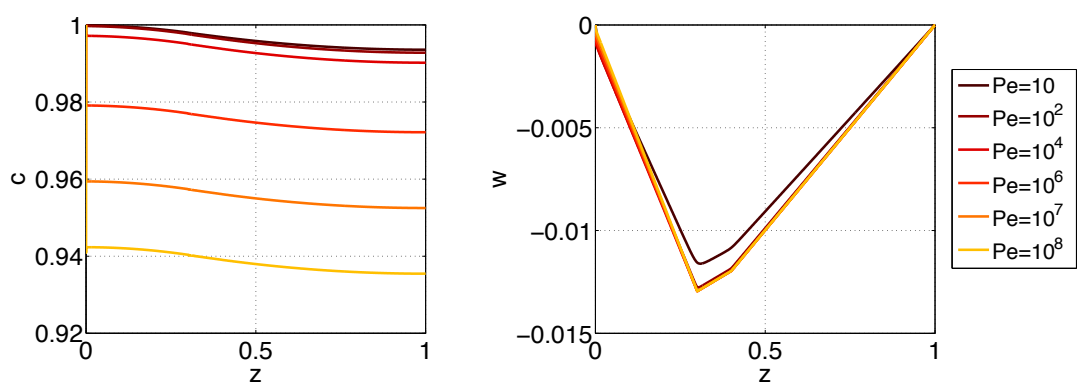

(b) Passive unloading, $\eta=(0,-0.01-0.02), \sigma=(0.04,0,0)$
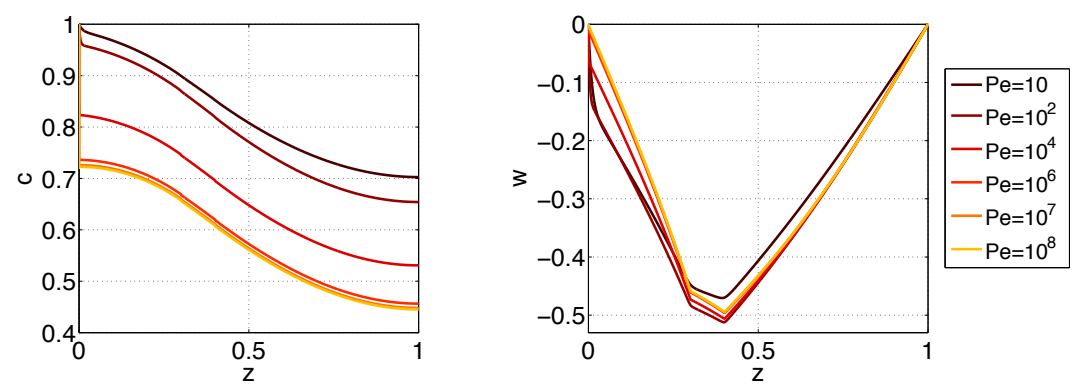

(c) Passive unloading, $\eta=(0,0-1), \sigma=(1,0,0)$

Figure 5: Profiles of phloem concentration, $c$, and velocity, $w$, against axial distance, $z$ for increasing $P e$, for $M=10^{3}, H=1, z_{1}=0.3, z_{2}=0.4$, $f=0$. 
The justification for this is that, for linear systems such as the flow within the xylem (which also contains boundary layers), the solution for concentration tends to a limit as $P e$ increases (as long as $f$ is zero). However this is not the case for the nonlinear flow considered here. For active unloading the solutions do not tend to a limit. Conversely for passive unloading, the solutions tend to a limit for large $F$. This behaviour is shown in Figure 5 which covers a range of $P e$ that encompasses the values for wheat $\left(P e=10^{4}\right)$ and black locust $\left(P e=10^{6}\right)$, and also includes higher values up to $P e=10^{8}$. For active unloading as shown in Figure 5(a), as $P e$ is increased, we can see that neither $c$ nor $w$ tend to a limit, and in fact the solutions diverge with $P e$. The profiles of $w$ do initially appear to converge for increasing $P e$, but then diverge, and the $P e$ for which the divergence occurs varies with $F$. Conversely, for passive unloading (Figures 5(b) and 5(c)), increasing $P e$ causes the profiles of $c$ and $w$ to converge.

The difference observed between the active and passive cases is due to the self limiting behaviour of the passive case. Integrating Equation (9) from $z=0$ to $z=1$ we obtain

$$
\left.\frac{d c}{d z}\right|_{z=0}=\left(\eta \int_{0}^{1} c d z-\sigma-f\right) P e
$$

As $P e$ increases, the limit of $(42)$, in the case of active loading $(\eta=0)$ is $\left.\frac{d c}{d z}\right|_{z=0}=\infty$. Conversely, in the case of passive loading $(\eta \neq 0)$, the limit of $\left.\frac{d c}{d z}\right|_{z=0}$ is bounded since $\int_{0}^{1} c d z$ can adjust to balance $-\sigma-f$.

In the passive case, the speed at which the profiles converge, with increasing $P e$, depends highly on the magnitude of $F$. For low values of $F$ (Figure 5(b)), $c$ has not yet converged at $P e=10^{8}$. Conversely for high values of $F$ (Figure 5(c)) both $c$ and $w$ have nearly converged at $P e=10^{8}$. To characterise this convergence we plot the ratio $R_{P e}$, which is defined as $c$ at $P e=10^{7}$ divided by $c$ at $P e=10^{6}$, against increasing loading and unloading for increasing values of $M$ in Figure 6 . Note that the loading is set to be active in the leaf zone and the unloading passive in the root zone such that $\sigma=(\tau, 0,0)$ and $\eta=(0,0,-\tau)$. For very small magnitudes of loading and unloading, there is only a small deviation from $c=1$ such that $R_{P e}$ is also very close to 1 . As $\tau$ increases, $R_{P e}$ at first decreases, indicating that the solution has not yet converged, and then slowly increases. For large $M$, convergence only occurs when the magnitude of the loading is $O(1)$.

Increasing $M$ reduces the deviation of $c$ away from $c=1$ and thus dampens the influence of the unloading terms and hence decreases the rate of convergence. Therefore, the validity of the limit $P e=\infty$ is strongly dependent on the parameter values of the system, which are biologically unknown. Hence, to improve accuracy of flow modelling in the phloem, the effects of diffusion must be included. 


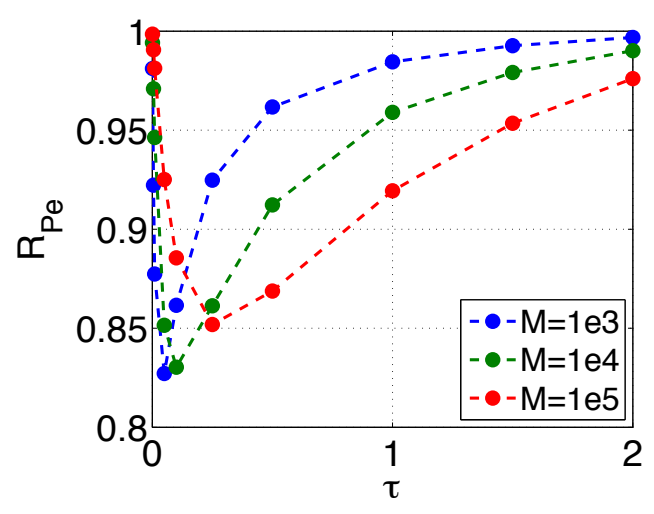

Figure 6: The ratio of $c$ at $P e=10^{7}$ over $P e=10^{6}, R_{P e}$, for increasing values of loading and unloading for three different values of $M$. The loading in the leaf zone is active and the unloading in the root zone is passive, $\sigma=(\tau, 0,0), \eta=(0,0,-\tau)$.

\subsection{Dependence of solution on $M$}

We now consider the effect of $M$ on the transport within the phloem. $M$ is the ratio of radial to axial conductivity and appears in Equation (8) which balances axial flow with radial flow. When the radial conductivity of the wall is zero, $M=0$, and hence no radial or axial flow occurs;

$$
\frac{d^{2} w}{d z^{2}}=0, \quad \text { subject to } w=0 \text { at } z=0 \text { and } z=1,
$$

such that $w=0$ everywhere. Therefore, no water flow occurs and transport is by diffusion only. When $M=\infty$, the system is in a state of osmotic equilibrium; $p=H c$ and $w=H \frac{d c}{d z}$. Hence, axial flow occurs which can transport $c$ down the phloem at a higher speed than diffusion alone.

The behaviour of the system as $M$ increases depends on the type and magnitude of loading. For low values of loading, the active and passive systems are similar, whereas for high values of loading they differ. An example of $c$ and $w$ for low values of loading is shown in Figure 7(a). For both active and passive systems, increasing $M$ increases the transport of $c$, reduces $\frac{d c}{d z}$, and increases the magnitude of $w$ to a limit.

Whilst the passive system has the same dynamics of flow for all $\eta$, the active system behaves differently for larger values of $\sigma$. Figures $7(\mathrm{~b})$ and $7(\mathrm{c})$ provide examples of the active and passive system respectively, for equal magnitudes of the unloading parameters: $\left(\sigma=\left(0,0,-2 \times 10^{-3}\right)\right.$ and $\eta=(0,0,0)$ in the active case; and $\sigma=(0,0,0), \eta=\left(0,0,-2 \times 10^{-3}\right)$ in the passive case). For the active case we see that as $M$ increases, $w$ increases then decreases towards to the limit, whereas in the passive case $w$ increases towards to the limit as before. 

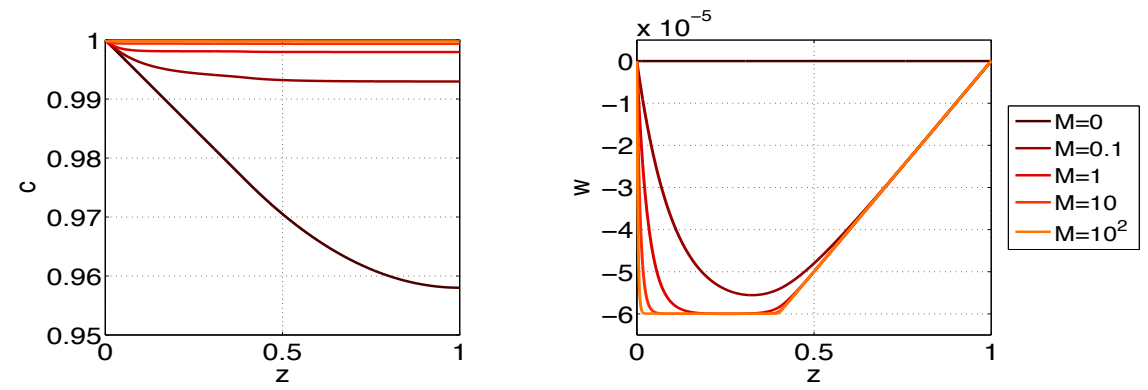

(a) Active unloading, $\sigma=\left(0,0,-1 \times 10^{-4}\right), \eta=(0,0,0)$
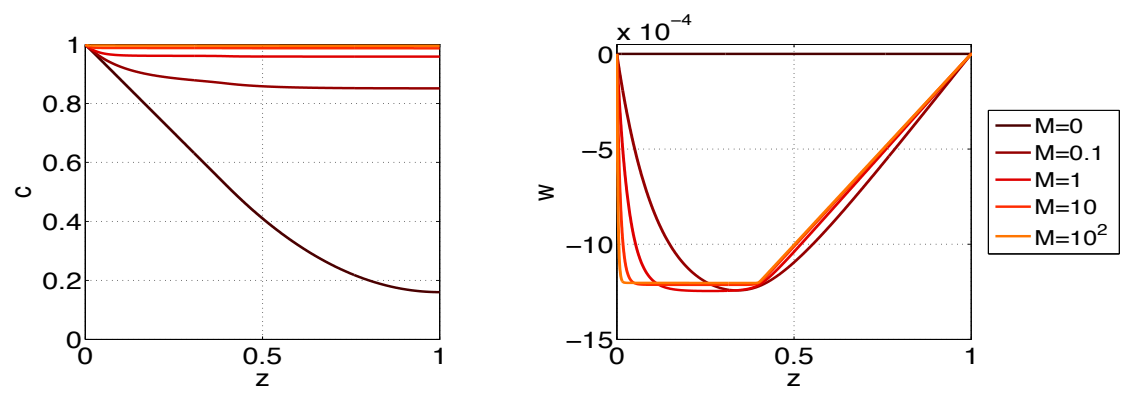

(b) Active unloading, $\sigma=\left(0,0,-2 \times 10^{-3}\right), \eta=(0,0,0)$
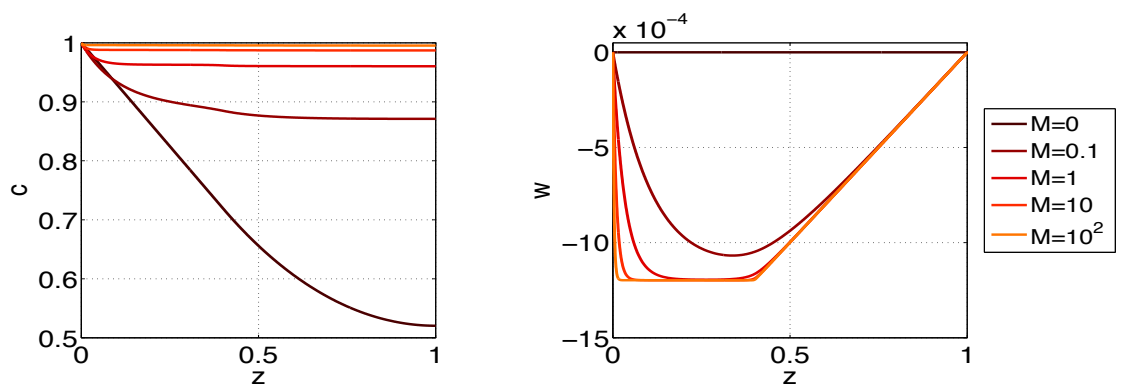

(c) Passive unloading, $\sigma=(0,0,0), \eta=\left(0,0,-2 \times 10^{-3}\right)$
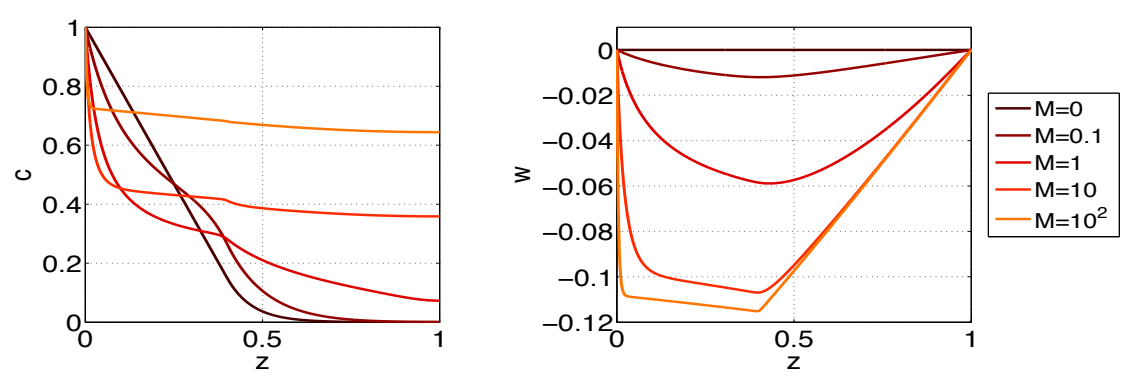

(d) Passive unloading, $\sigma=(0,0,0), \eta=\left(0,0,-2 \times 10^{-1}\right)$

Figure 7: Profiles of phloem concentration, $c$, and velocity, $w$ against axial distance, $z$ for increasing $M$, for $P e=10^{3}, H=1, z_{1}=0.3, z_{2}=0.4$, $f=0$. 
The difference between the two cases is because the unloading of $c$ is larger in the active case than for the passive case. The unloading in the passive case is limited since unloading is proportional to $c$. The large unloading in the active case gives rise to an axial velocity which is larger in magnitude than the high- $M$ limit. Whereas in the passive system, the limited loading causes the axial velocity to be lower in magnitude than the limit.

For even higher unloading parameters, the active system quickly becomes unphysical since unloading irrespective of $c$ results in negative values for $c$. For the passive system instead, in Figure $7(\mathrm{~d})$ we see a larger $\frac{d c}{d z}$ caused by the larger unloading, and that $w$ again increases in magnitude towards a limit, but the approach to the limit has slowed.

\subsection{Dependence of solution on $f$}

The inclusion of diffusion also permits the modelling of an axial efflux at the terminus of the phloem in the root. Figure 8 shows the profiles of $c$ and $w$ against $z$ comparing the analytical to the numerical solution for $M=10^{4}$, $P e=10^{5}, H=1, \eta=(0,-0.01,-0.02), \sigma=(0.04,0,0)$ for increasing values of $f$. For these parameter values, the analytical and numerical solution

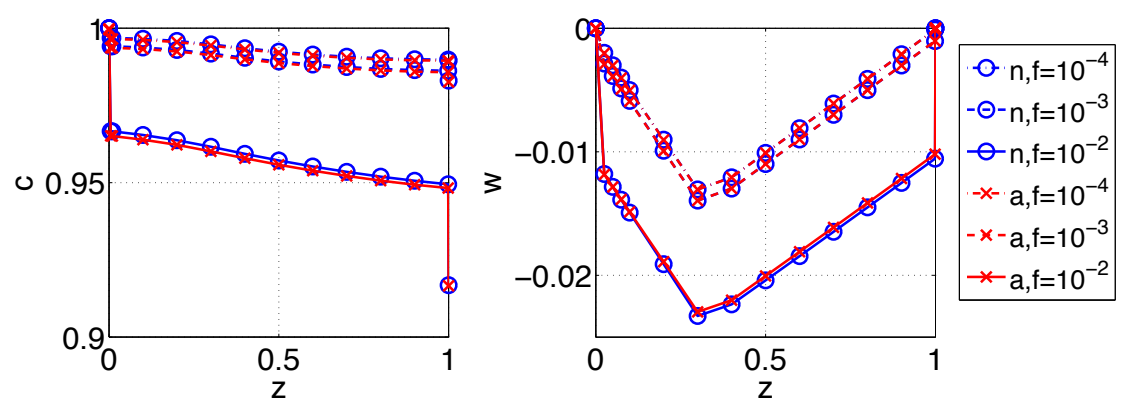

Figure 8: Profiles of phloem concentration, $c$, and velocity, $w$, against axial distance, $z$, comparing the numerical solution, $n$, to the analytical solution, a, for varying axial effluxes, $f$, for $M=10^{4}$. Pe $=10^{5}$, $H=1, z_{1}=0.3, z_{2}=0.4, \phi=1, \eta=(0,-0.01-0.02)$, $\sigma=(0.04,0,0)$.

agree well, and we can see that an efflux of sugar at $z=1$ now gives rise to a boundary layer in both $c$ and $w$ at $z=1$. The boundary layer significantly influences the solutions in the bulk. As $f$ increases, the solution of $c$ decreases in magnitude, and $w$ increases in magnitude, not just near $z=1$ but for all $z$. The steepness of the boundary layer increases with the magnitude of $f$ since $\frac{d c^{R}}{d z}=-f P e$ at $z=1$. Extremely steep boundary layers are unphysical, therefore $f$ is unlikely to be much greater than $O(1 / P e)$. 


\section{Discussion}

We have presented a mathematical model for the transport of sugar and water within a phloem vessel in response to an osmotically-generated pressure gradient. The magnitude and type of sugar loading and unloading are unknown. Therefore, we have considered active and passive loading and unloading. Passive loading represents the transport of sugar down the sugar concentration gradient whilst active loading represents transport via a pump.

We have solved the system numerically for all parameter values, and analytically in the limit of high Münch number using the method of matched asymptotic expansions. We have seen that the analytical solution compares well to the numerical solution for both black locust and wheat parameter values when the unloading is active, and less well when the unloading is passive and $M$ is small. Thompson \& Holbrook (2003a) expect that most plants fall within the osmotic equilibrium regime i.e. high $M$ for which the asymptotic solution is valid.

Due to the high $P e$ number of sugar transport, the majority of previous authors neglect the effects of diffusion entirely. One of the primary motivations for this paper was to analyse whether this assumption is valid. Diffusion can only be neglected if the solutions become independent of $\mathrm{Pe}$ at $P e$ numbers typical for plants (for example, as shown in Figure 5(c)). We have found that the boundary layer terms drive the solutions in the bulk. The boundary layer terms arise due to the fact that diffusion dominates at the boundaries. Consequently, the effects of diffusion are important not only in the boundary layer but for the entire length of the phloem. Since the solutions derive mainly from the boundary conditions, it is difficult to compare our results to previous work where diffusion is neglected. For example, Jensen et al. (2011, 2012) consider a convection-only system, which reduces the order of the governing equations, and hence apply the boundary conditions that $c$ is finite at the vessel ends. Pickard \& Abraham-Shrauner (2009) do include diffusion in their analysis, but only consider the translocation zone, and axial loading only. They also neglect the longitudinal variation of pressure, which is equivalent to considering the limit that $H \gg 1$. This therefore places the solution in a different parameter regime to that considered in this paper, and therefore makes it difficult to compare the features of the solutions.

Plotting the numerical solution for increasing $P e$ we found the system behaved differently for active and passive loading. For active unloading, the solutions diverge with increasing $P e$. Conversely for passive unloading, which is more physical for low sugar concentration, the solutions converge. However, the rate of convergence, for $P e$ values typical for a plant, depends on the magnitude of loading and unloading. For low magnitude loading the solutions have not yet converged in $P e$ for $P e$ typical of plants, and therefore 
cannot be approximated by $\mathrm{Pe} \rightarrow \infty$ solutions, whereas for $O(1)$ loading, convergence occurs. The difference between the active and passive systems is that unloading is unbounded in the active case, and bounded in the passive case. In effect, the passive case self-limits the unloading. Therefore, the validity of the assumption that diffusion can be neglected entirely depends on the type and magnitude of loading, which is unknown.

We also considered the effect of increasing $M$ on the system. For low magnitude loading and unloading, the active and passive cases behaved similarly, and increasing $M$ increased the transport of $c$ and increased the magnitude of $w$ to a limit. For higher magnitude loading the active system behaved differently, causing the axial velocity to increase and then decrease towards the high- $M$ limit. Again the difference between the active and passive systems is due to self-limiting behaviour of the passive case.

The inclusion of diffusion also allows the modelling of an axial efflux which may occur to transport sugar to the growing meristem or as a sugar exudate to promote bacterial interactions. This flux has not been previously observed, but experiments, similar to those by Dilkes et al. (2004) that measure the profile of carbon within the phloem, should be carried out to see if boundary layer effects occur at the phloem tip which could provide an estimate for the magnitude of $f$.

For simplicity we have assumed that the osmotic potential is a linear function of concentration and that viscosity is constant. However, Thompson \& Holbrook (2003a) and Jensen et al. (2012) argue that this is not likely to be the case for the large concentration of sugar in the phloem. In addition, we have assumed that the phloem is in isolation, whereas it is actually in close proximity to the xylem, which influences the dynamics of transport (Hölttä, 2006; Lacointe \& Minchin, 2008; Hall \& Minchin, 2013). Therefore, future work should include these effects.

In summary, this work highlights the role of diffusion in sugar transport within the phloem. Due to the biological uncertainty regarding the nature of sugar loading and unloading, ignoring diffusion effects might lead to erroneous results. Therefore, to improve the accuracy of phloem modelling, diffusion should be included and further experiments should be carried out to determine the mechanisms and magnitudes of sugar loading and unloading.

\section{Acknowledgements}

This work was sponsored by Defra, BBSRC (BB/J000868/1), Scottish Government, AHDB, and other industry partners through Sustainable Arable LINK Project LK09136 and the BBSRC (BB/I024283/1). Tiina Roose is funded by a Royal Society University Research Fellowship. 


\section{References}

C. Barbaroux, N. Bréda, and E. Dufrêne, (2003), Distribution of aboveground and below-ground carbohydrate reserves in adult trees of two contrasting broad-leaved species (Quercus petraea and Fagus sylvatica), New Phytologist 157(3), 605-615.

I. J. Bingham, and E. A. Stevenson, (1993), Control of root growth: effects of carbohydrates on the extension, branching and rate of respiration of different fractions of wheat roots, Physiologia Plantarum 88(1), 149-158.

V. De Schepper, T. De Swaef, I. Bauwerarts, and K. Steppe, (2013), Phloem transport: a review of mechanisms and controls, J. Exp. Bot. 64(16), 48394850 .

N. B. Dilkes. D. L. Jones, and J. Farrar, (2004), Temporal dynamics of carbon partitioning and rhizodeposition in wheat, Plant Physiology 134(2), 706-715.

V. Fernández, and P. H. Brown, (2013), From plant surface to plant metabolism: the uncertain fate of foliar-applied nutrients, Frontiers in plant science $4(289)$.

A. J. Hall and P. E. H. Minchin, (2013), A closed-form solution for steadystate coupled phloem/xylem flow using the Lambert-W function, Plant, cell $\mathcal{E}$ environ. 36(12), 2150-2162.

E. J. Hinch, (1991), Perturbation methods Vol(6), Cambridge University Press, Cambridge.

T. Hölttä, T. Vesala, S. Sevanto, M. Perämäki, E. Nikinmaa, (2006), Modeling xylem and phloem water flows in trees according to cohesion theory and Münch hypothesis, Trees 20(1), 67-78.

K. H. Jensen, E. Rio, R. Hansen, C. Clanet, and T. Bohr, (2009), Osmotically driven pipe flows and their relation to sugar transport in plants, $J$. Fluid Mech. 636, 371-396.

K. H. Jensen, J. Lee, T. Bohr, H. Bruus, N. M. Holbrook, and M. A, Zwieniecki, (2011), Optimality of the Münch mechanism for translocation of sugars in plants, J. R. Soc. Interface 8(61), 1155-1165.

K. H. Jensen, K. Berg-Sørensen, S. M. Friis, and T. Bohr, (2012), Analytic solutions and universal properties of sugar loading models in Münch phloem flow, J. Theor. Biol. 304, 286-296.

K. H. Jensen, J. A, Savage, and N. M. Holbrook, (2013), Journal of The Royal Society Interface, 10(83), 20130055. 
P. J. Kramer and J. S. Boyer, (1995), Water relations of plants and soils, Academic Press, San Diego, Chapter 2, pg 38.

L. Kutschera, E. Lichtenegger, and M. Sobotik, (2009), Wurzelatlas der Kulturpflanzen gemigter Gebiete mit Arten des Feldgemsebaues DLG Verlag, Frankfurt, pg 228.

A. Lacointe and P. E. H. Minchin, (2008), Modelling phloem and xylem transport within a complex architecture, Functional Plant Biology 35(10), 772-780.

P. E. H. Minchin, and M. R. Thorpe, (1987), Measurement of unloading and reloading of photo-assimilate within the stem of bean, J. Exp. Bot. $38(117), 211-220$.

P. E. H. Minchin, and M. R. Thorpe, (1996), What determines carbon partitioning between competing sinks?, J. Exp. Bot. 47, 1293-1296.

E. Münch, (1926), Über Dynamik der Saftströmungen, Deut. Bot. Ges. 44, 68-71.

J. W. Patrick, (1997), Phloem unloading: sieve element unloading and postsieve element transport, Annu. Rev. Plant Physiol. 48(1), 191-222.

S. Payvandi, K. R. Daly, D. L. Jones, P. Talboys, K. C. Zygalakis, T. Roose, (2014), A mathematical model of water and nutrient transport in xylem vessels of a wheat plant, Bull. Math. Biol. 76(3), 566-596.

R. J. Phillips, and S. R. Dungan, (1993), Asymptotic analysis of flow in sieve tubes with semi-permeable walls. J. Theor. Biol, 162(4), 465-485.

W. F. Pickard, and B. Abraham-Shrauner, (2009). A simplest steady-state Münch-like model of phloem translocation, with source and pathway and sink, Functional Plant Biology 36(7), 629-644.

H. M. Rawson and L. T. Evans, (1971), The contribution of stem reserves to grain development in a range of wheat cultivars of different height, Aust. J. Agric. Res. 22(6), 851-863.

M. V. Thompson, and N. M. Holbrook, (2003a), Application of a singlesolute non-steady-state phloem model to the study of long-distance assimilate transport, J. Theor. Biol, 220(4), 419-455.

M. V. Thompson, and N. M. Holbrook, (2003b), Scaling phloem transport: water potential equilibrium and osmoregulatory flow, Plant, Cell $\&$ Environment 26(9), 1561-1577.

M. V. Thompson, and N. M. Holbrook, (2004), Scaling phloem transport: information transmission, Plant, Cell ES Environment 27(4), 509-519. 
M. V. Thompson, (2005), Scaling phloem transport: Elasticity and pressureconcentration waves, J. Theor. Biol, 236(3), 229-241.

R. Turgeon, (2010), The role of phloem loading reconsidered, Plant Physiology 152(4), 1817-1823.

A. J. E. van Bel, (2003), The phloem, a miracle of ingenuity. Plant, Cell and Environment 26(1), 125-149.

\section{Appendix A: Scaled equations and boundary con- ditions}

The scaled fluid transport and concentration transport equations are

$$
\begin{aligned}
& \epsilon^{1+\delta-\beta} \frac{d^{2} \bar{w}^{i}}{d z^{2}}=\epsilon^{\delta-\beta} \bar{w}^{i}-\bar{H} \frac{d c^{i}}{d z}, \\
& \epsilon^{\delta-\chi} \frac{d c^{i}}{d z} \bar{w}^{i}+\epsilon^{\delta-\chi} c^{i} \frac{d \bar{w}^{i}}{d z}+\frac{1}{\bar{P} e} \frac{d^{2} c^{i}}{d z^{2}}=-\epsilon^{\gamma-\chi} \bar{F}^{i},
\end{aligned}
$$

in the bulk regions. In the leaf, stem, and root zones, the left-hand-side boundaries are $z=0, z=z_{1}$, and $z=z_{2}$ respectively, and we rewrite $z$ in terms of a boundary layer variable $x^{i}$ as; $z=\epsilon^{\alpha} x^{L}$ in the leaf, $z=z_{1}+\epsilon^{\alpha} x^{S}$ in the stem, and $z=z_{2}+\epsilon^{\alpha} x^{R}$ in the root. Therefore, the equations in the LHS boundary region are

$$
\begin{aligned}
& \epsilon^{1-\alpha+\delta-\beta} \frac{d^{2} \bar{w}^{i}}{d x^{i 2}}=\epsilon^{\delta+\alpha-\beta} \bar{w}^{i}-\bar{H} \frac{d c^{i}}{d x^{i}}, \\
& \epsilon^{\alpha+\delta-\chi} \frac{d c^{i}}{d x^{i}} \bar{w}^{i}+\epsilon^{\alpha+\delta-\chi} c^{i} \frac{d \bar{w}^{i}}{d x^{i}}+\frac{1}{\bar{P} e} \frac{d^{2} c^{i}}{d x^{i 2}}=-\epsilon^{\gamma+2 \alpha-\chi} \bar{F}^{i} .
\end{aligned}
$$

In the leaf, stem, and root zones, the right-hand-side boundaries respectively are $z=z_{1}, z=z_{2}$, and $z=1$, and we rewrite $z$ in terms of a boundary layer variable $y^{i}$ as; $z=z_{1}-\epsilon^{\alpha} y^{L}$ in the leaf, $z=z_{2}-\epsilon^{\alpha} y^{S}$ in the stem, and $z=1-\epsilon^{\alpha} y^{R}$ in the root. Therefore, the RHS boundary region equations are

$$
\begin{aligned}
& \epsilon^{1-\alpha+\delta-\beta} \frac{d^{2} \bar{w}^{i}}{d y^{i 2}}=\epsilon^{\delta+\alpha-\beta} \bar{w}^{i}+\bar{H} \frac{d c^{i}}{d y^{i}}, \\
& -\epsilon^{\alpha+\delta-\chi} \frac{d c^{i}}{d y^{i}} \bar{w}^{i}-\epsilon^{\alpha+\delta-\chi} c^{i} \frac{d \bar{w}^{i}}{d y^{i}}+\frac{1}{\bar{P} e} \frac{d^{2} c^{i}}{d y^{i, 2}}=-\epsilon^{\gamma+2 \alpha-\chi} \bar{F}^{i} .
\end{aligned}
$$

The boundary conditions at $z=0$ and $z=1$ become

$$
\begin{gathered}
\bar{w}^{L}=0, \quad c^{L}=\phi, \quad \text { at } \quad x^{L}=0, \\
\bar{w}^{R}=0, \quad \frac{d c^{R}}{d y^{R}}=\epsilon^{\gamma+\alpha-\chi} \bar{P} e \bar{f} \quad \text { at } \quad y^{R}=0 .
\end{gathered}
$$


The continuity conditions at the leaf-stem boundary are

$$
\begin{gathered}
c^{L}=c^{S}, \quad \epsilon^{\delta} \bar{w}^{L}=\epsilon^{\delta} \bar{w}^{S}, \\
\epsilon^{\delta} c^{L} \bar{w}^{L}+\frac{\epsilon^{\chi}}{\bar{P} e} \frac{d c^{L}}{d z}=\epsilon^{\delta} c^{S} \bar{w}^{S}+\frac{\epsilon^{\chi}}{\bar{P} e} \frac{d c^{S}}{d z}, \quad \epsilon^{\delta} \frac{d \bar{w}^{L}}{d z}=\epsilon^{\delta} \frac{d \bar{w}^{S}}{d z} .
\end{gathered}
$$

The continuity conditions at the stem-root boundary are

$$
\begin{gathered}
c^{S}=c^{R}, \quad \epsilon^{\delta} \bar{w}^{S}=\epsilon^{\delta} \bar{w}^{R}, \\
\epsilon^{\delta} c^{S} \bar{w}^{S}+\frac{\epsilon^{\chi}}{\bar{P} e} \frac{d c^{S}}{d z}=\epsilon^{\delta} c^{R} \bar{w}^{R}+\frac{\epsilon^{\chi}}{\bar{P} e} \frac{d c^{R}}{d z}, \quad \epsilon^{\delta} \frac{d \bar{w}^{S}}{d z}=\epsilon^{\delta} \frac{d \bar{w}^{R}}{d z} .
\end{gathered}
$$

\section{Appendix B: Analytical solution procedure}

Here we give the procedure to determine $c^{i}$ and $\bar{w}^{i}$ at $O\left(\epsilon^{0}\right)$ and $O\left(\epsilon^{\frac{1}{2}}\right)$. The overall method involves solving the fluid transport and concentration transport equations in each region, applying the boundary conditions at $z=$ 0 and $z=1$, matching the solutions across the regions to obtain composite solutions in each zone, and finally applying continuity conditions at $z=z_{1}$ and $z=z_{2}$ to fully determine the solutions. We start at Region 1 which is in the leaf zone.

\section{B.1 Region 1}

This region represents the LHS boundary layer in the leaf zone. The solution in Region 1 has to match to the solution in Region 2 and also satisfy the boundary conditions

$$
\begin{aligned}
c_{0}^{L}+\epsilon^{\frac{1}{2}} c_{1}^{L}+\epsilon c_{2}^{L}+O\left(\epsilon^{\frac{3}{2}}\right)=\phi, & \text { at } x^{L}=0 \\
\bar{w}_{0}^{L}+\epsilon^{\frac{1}{2}} \bar{w}_{1}^{L}+\epsilon \bar{w}_{2}^{L}+O\left(\epsilon^{\frac{3}{2}}\right)=0, & \text { at } x^{L}=0 .
\end{aligned}
$$

The $O\left(\epsilon^{0}\right)$ terms of equations (21) and (22) are

$$
-\bar{H} \frac{d c_{0}^{L}}{d x^{L}}=0, \quad \frac{1}{\bar{P} e} \frac{d^{2} c_{0}^{L}}{d x^{L 2}}=0,
$$

and applying the boundary condition (B.1), the solution for $c_{0}^{L}$ in Region 1 is $c_{0}^{L}=\phi$. The $O\left(\epsilon^{\frac{1}{2}}\right)$ terms of equations are (21) and (22) are

$$
-\bar{H} \frac{d c_{1}^{L}}{d x^{L}}=0, \quad \frac{1}{\bar{P} e} \frac{d^{2} c_{1}^{L}}{d x^{L 2}}=0,
$$

and applying the boundary condition (B.1), the solution for $c_{1}^{L}$ in Region 1 is $c_{1}^{L}=0$. The $O(\epsilon)$ terms of equations (21) and (22) are

$$
\frac{d^{2} \bar{w}_{0}^{L}}{d x^{L 2}}=\bar{w}_{0}^{L}-\bar{H} \frac{d c_{2}^{L}}{d x^{L}}
$$




$$
\frac{d c_{0}^{L}}{d x^{L}} \bar{w}_{0}^{L}+c_{0}^{L} \frac{d \bar{w}_{0}^{L}}{d x^{L}}+\frac{1}{\bar{P} e} \frac{d^{2} c_{2}^{L}}{d x^{L 2}}=0
$$

and substituting $c_{0}^{L}=\phi$ into (B.6) and integrating, we obtain $\frac{d c_{2}^{L}}{d x^{L}}=$ $-\bar{P} e \phi \bar{w}_{0}^{L}+k_{1}$ where $k_{1}$ is an unknown constant. Substituting back into (B.5) yields

$$
\frac{d^{2} \bar{w}_{0}^{L}}{d x^{L 2}}=\bar{w}_{0}^{L}(1+\bar{H} \bar{P} e \phi)-\bar{H} k_{1} .
$$

Solving (B.7), applying the boundary condition (B.2), and neglecting any exponentially growing terms, we obtain

$$
\bar{w}_{0}^{L}=\frac{k_{1} \bar{H}}{1+\bar{H} \bar{P} e \phi}\left(1-e^{-\sqrt{1+\bar{H} \bar{P} e \phi} x^{L}}\right) .
$$

The constant $k_{1}$ can only be determined by applying continuity conditions between the plant zones but this can only occur once the composite solution is determined for the entirety of the leaf zone. We therefore proceed to Region 2 where we repeat this solution procedure.

\section{B.2 Region 2}

This region represents the central bulk of the leaf zone and the solution in this region has to match to both the solution in Region 1 and Region 3. The $O\left(\epsilon^{0}\right)$ terms of equations (19) and (20) are

$$
-\bar{H} \frac{d c_{0}^{L}}{d z}=0, \quad \frac{1}{\bar{P} e} \frac{d^{2} c_{0}^{L}}{d z^{2}}=0
$$

such that $c_{0}^{L}$ in Region 2 is a constant, which upon matching to Region 1 gives $c_{0}^{L}=\phi$. The $O\left(\epsilon^{\frac{1}{2}}\right)$ terms of equations (19) and (20) are

$$
0=\bar{w}_{0}^{L}-\bar{H} \frac{d c_{1}^{L}}{d z}, \quad \frac{d c_{0}^{L}}{d z} \bar{w}_{0}^{L}+c_{0}^{L} \frac{d \bar{w}_{0}^{L}}{d z}+\frac{1}{\overline{P e}} \frac{d^{2} c_{1}^{L}}{d z^{2}}=-\bar{F}_{0}^{L},
$$

where $\bar{F}_{0}^{L}=\bar{\eta}^{L} c_{0}^{L}+\bar{\sigma}^{L}$. Substituting $c_{0}^{L}=\phi$, solving Equation (B.10), and matching to the solution in Region 1, we find

$$
\begin{aligned}
c_{1}^{L} & =\frac{\bar{P} e}{\bar{H} \bar{P} e \phi+1}\left(-\bar{F}_{0}^{L} \frac{z^{2}}{2}+\frac{k_{1}}{\bar{P} e} z\right), \\
\bar{w}_{0}^{L} & =\frac{\bar{H} \bar{P} e}{\bar{H} \bar{P} e \phi+1}\left(-\bar{F}_{0}^{L} z+\frac{k_{1}}{\bar{P} e}\right) .
\end{aligned}
$$




\section{B.3 Region 3}

This region represents the RHS boundary layer in the leaf zone. The solution in Region 3 has to match to the solution in Region 2 and satisfy continuity conditions at $z=z_{1}$. The $O\left(\epsilon^{0}\right)$ terms of equations (23) and (24) are

$$
\bar{H} \frac{d c_{0}^{L}}{d y^{L}}=0, \quad \frac{1}{\bar{P} e} \frac{d^{2} c_{0}^{L}}{d y^{L 2}}=0,
$$

such that $c_{0}^{L}$ in Region 3 is a constant, which upon matching to Region 2 gives $c_{0}^{L}=\phi$. The $O\left(\epsilon^{\frac{1}{2}}\right)$ terms of equations (23) and (24) are

$$
\bar{H} \frac{d c_{1}^{L}}{d y^{L}}=0, \quad \frac{1}{\overline{P e}} \frac{d^{2} c_{1}^{L}}{d y^{L 2}}=0,
$$

which indicates that $c_{1}^{L}$ in Region 3 is a constant and we let $c_{1}^{L}=B_{1}^{L}$. The $O(\epsilon)$ terms of equations (23) and (24) are

$$
\begin{aligned}
& \frac{d^{2} \bar{w}_{0}^{L}}{d y^{L 2}}=\bar{w}_{0}^{L}+\bar{H} \frac{d c_{2}^{L}}{d y^{L}}, \\
- & \frac{d c_{0}^{L}}{d y^{L}} \bar{w}_{0}^{L}-c_{0}^{L} \frac{d \bar{w}_{0}^{L}}{d y^{L}}+\frac{1}{\bar{P} e} \frac{d^{2} c_{2}^{L}}{d y^{L 2}}=0,
\end{aligned}
$$

and substituting $c_{0}^{L}=\phi$ into (B.16) and integrating, we obtain $\frac{d c_{2}^{L}}{d y^{L}}=$ $-\bar{P} e \phi \bar{w}_{0}^{L}+B_{2}^{L}$ where $B_{2}^{L}$ is an unknown constant. Substituting back into (B.15) yields

$$
\frac{d^{2} \bar{w}_{0}^{L}}{d y^{L 2}}=\bar{w}_{0}^{L}(1+\bar{H} \bar{P} e \phi)+\bar{H} B_{2}^{L} .
$$

Solving for $\bar{w}_{0}^{L}$ and neglecting any exponentially growing terms we obtain

$$
\bar{w}_{0}^{L}=A_{1} e^{-\sqrt{1+\bar{H} \bar{P} e \phi} y^{L}}-\frac{\bar{H} B_{2}^{L}}{\bar{H} \bar{P} e \phi+1} .
$$

Matching to Region 2 gives

$$
B_{1}^{L}=\frac{\bar{P} e}{\bar{H} \bar{P} e \phi+1}\left(-\frac{\bar{F}_{0}^{L} z_{1}^{2}}{2}+\frac{k_{1}}{\bar{P} e} z_{1}\right), \quad B_{2}^{L}=\bar{P} e \bar{F}_{0}^{L} z_{1}-k_{1} .
$$

Combining the solutions for $c$ and $\bar{w}$ for all three regions within the leaf zone we obtain the following composite solution for the leaf zone

$$
\begin{aligned}
c_{0}^{L} & =\phi, \\
c_{1}^{L} & =\frac{\bar{P} e}{\kappa}\left(-\bar{F}_{0}^{L} \frac{z^{2}}{2}+\frac{k_{1}}{\bar{P} e} z\right),
\end{aligned}
$$




$$
\bar{w}_{0}^{L}=\frac{\bar{H} \bar{P} e}{\kappa}\left(-\bar{F}_{0}^{L} z+\frac{k_{1}}{\bar{P} e}\left(1-e^{-\sqrt{\kappa} \epsilon^{-\frac{1}{2}} z}\right)\right)+A_{1} e^{-\sqrt{\kappa} \epsilon^{-\frac{1}{2}}\left(z_{1}-z\right)},
$$

where $\kappa=\bar{H} \bar{P} e \phi+1$ and where $k_{1}$ and $A_{1}$ are unknown constants to be determined by applying continuity conditions at the zone boundaries. Before continuity can be applied, we first calculate the stem and root composite solutions. For brevity, we do not show the details here, but we do use the same procedure outlined in Sections B.1 to B.3 for the leaf zone.

\section{B.4 Applying continuity conditions}

Solving at $O\left(\epsilon^{0}\right)$ in the remaining 6 regions we determine that $c_{0}$ is constant everywhere. Assuming that $c_{0}$ is continuous across the zone boundaries, we find that $c_{0}^{i}=\phi$ for all nine regions. Solving at the next order, $O\left(\epsilon^{\frac{1}{2}}\right)$, we find the following composite solutions in the stem and root zones

$$
\begin{aligned}
c_{1}^{S} & =\frac{\bar{P} e}{\kappa}\left(-\bar{F}_{0}^{S} \frac{z^{2}}{2}+k_{2} z+k_{3}\right), \\
\bar{w}_{0}^{S} & =\frac{\bar{H} \bar{P} e}{\kappa}\left(-\bar{F}_{0}^{S} z+k_{2}\right)+A_{2} e^{-\sqrt{\kappa} \epsilon^{-\frac{1}{2}}\left(z-z_{1}\right)}+A_{3} e^{-\sqrt{\kappa} \epsilon^{-\frac{1}{2}}\left(z_{2}-z\right),} \\
c_{1}^{R} & =\frac{\bar{P} e}{\kappa}\left(-\bar{F}_{0}^{R} \frac{z^{2}}{2}+\left(\bar{F}^{R}-\bar{f}\right) z+k_{4}\right), \\
\bar{w}_{0}^{R} & =\frac{\bar{H} \bar{P} e}{\kappa}\left(-\bar{F}_{0}^{R} z+\left(\bar{F}_{0}^{R}-\bar{f}\right)\right)+\frac{\bar{H} \bar{P} e \bar{f}}{\kappa} e^{-\sqrt{\kappa} \epsilon^{-\frac{1}{2}}(1-z)}+A_{4} e^{-\sqrt{\kappa} \epsilon^{-\frac{1}{2}}\left(z-z_{2}\right)},
\end{aligned}
$$

where $k_{1}, k_{2}, k_{3}, k_{4}, A_{1}, A_{2}, A_{3}, A_{4}$ are unknown constants to be determined from applying continuity boundary conditions.

As can be seen, the solutions for $\bar{w}_{0}^{i}$ contain exponential terms due to the boundary layer terms. This implies that terms of a higher order (for example $c_{2}^{i}$ and $\bar{w}_{1}{ }^{i}$ ) can contribute to the flux continuity conditions due to the presence of $\epsilon^{-\frac{1}{2}}$ within the exponential. For example if we write

$$
\bar{w}^{i}=\bar{w}^{i, b u l k}+\bar{w}^{i, e x p} e^{\epsilon^{-\frac{1}{2}} f(z)}, \quad c^{i}=c^{i, b u l k}+c^{i, e x p} e^{\epsilon^{-\frac{1}{2}} f(z)},
$$

then the derivatives, $\frac{d \bar{w}^{i}}{d z}$ and $\frac{d c^{i}}{d z}$, which appear in the flux continuity conditions become

$$
\begin{aligned}
\frac{d \bar{w}^{i}}{d z} & =\epsilon^{-\frac{1}{2}}\left(\frac{d f_{0}}{d z} \bar{w}_{0}^{i, \exp } e^{\epsilon^{-\frac{1}{2}} f_{0}}\right) \\
& +\epsilon^{0}\left(\frac{d \bar{w}_{0}^{i, b u l k}}{d z}+\frac{d \bar{w}_{0}^{i, e x p}}{d z} e^{\epsilon^{-\frac{1}{2}} f_{0}}+\frac{d f_{1}}{d z} \bar{w}_{1}^{i, \text { exp }} e^{\epsilon^{-\frac{1}{2}} f_{1}}\right)+O\left(\epsilon^{\frac{1}{2}}\right), \\
\frac{d c^{i}}{d z} & =\epsilon^{-\frac{1}{2}}\left(\frac{d f_{0}}{d z} c_{0}^{i, e x p} e^{\epsilon^{-\frac{1}{2}} f_{0}}\right)
\end{aligned}
$$




$$
+\epsilon^{0}\left(\frac{d c_{0}^{i, b u l k}}{d z}+\frac{d c_{0}^{i, e x p}}{d z} e^{\epsilon^{-\frac{1}{2}} f_{0}}+\frac{d f_{1}}{d z} c_{1}^{i, \exp } e^{\epsilon^{-\frac{1}{2}} f_{1}}\right)+O\left(\epsilon^{\frac{1}{2}}\right) .
$$

Since $c_{0}^{i, e x p}=c_{1}^{i, e x p}=0$ and $\frac{d c_{0}^{i, b u l k}}{d z}=0$, the first contribution to $\frac{d c^{i}}{d z}$ is at $O\left(\epsilon^{\frac{1}{2}}\right)$. The consequence of this is that the exponential terms of $c_{2}^{i}$ first have to be calculated before continuity conditions can be applied and $c_{1}^{i}$ and $\bar{w}_{0}^{i}$ can be fully determined.

Solving for $c_{2}^{i}$ in all the Regions using the same procedure as in Sections B.1 to B.3, and then applying the continuity conditions we find

$$
\begin{gathered}
k_{1}=\bar{P} e\left(z_{1}\left(\bar{F}_{0}^{L}-\bar{F}_{0}^{S}\right)+z_{2}\left(\bar{F}_{0}^{S}-\bar{F}_{0}^{R}\right)+\bar{F}_{0}^{R}-\bar{f}\right), \\
k_{2}=z_{2}\left(\bar{F}_{0}^{S}-\bar{F}_{0}^{R}\right)+\bar{F}_{0}^{R}-\bar{f}, \\
k_{3}=\frac{z_{1}^{2}}{2}\left(\bar{F}_{0}^{L}-\bar{F}_{0}^{S}\right), \\
k_{4}=\frac{z_{2}^{2}}{2}\left(\bar{F}_{0}^{S}-\bar{F}_{0}^{R}\right)+\frac{z_{1}^{2}}{2}\left(\bar{F}_{0}^{L}-\bar{F}_{0}^{S}\right), \\
A_{1}=A_{2}=A_{3}=A_{4}=0,
\end{gathered}
$$

indicating that, at this order, there are no boundary layer terms near $z_{1}$ and $z_{2}$. Finally, we calculate the exponential terms of $c_{3}^{i}$ in all Regions to fully determine $c_{2}^{i}$ and $\bar{w}_{1}^{i}$, the solutions of which are given in Appendix C. At this order, we find that boundary layer terms are non-zero at $z_{1}$ and $z_{2}$ and hence we terminate the calculation here.

\section{Appendix C: Analytical solution}

Here, we provide the solutions for $c_{0}^{i}, c_{1}^{i}, c_{2}^{i}, c_{3}^{i} \bar{w}_{0}^{i}$ and $\bar{w}_{1}^{i}$

$$
\begin{aligned}
c_{0}^{L} & =c_{0}^{S}=c_{0}^{R}=\phi \\
c_{1}^{L} & =\frac{\bar{P} e}{\kappa}\left(-\bar{F}_{0}^{L} \frac{z^{2}}{2}+\frac{k_{1}}{\bar{P} e} z\right) \\
c_{1}^{S} & =\frac{\bar{P} e}{\kappa}\left(-\bar{F}_{0}^{S} \frac{z^{2}}{2}+k_{2} z+k_{3}\right) \\
c_{1}^{R} & =\frac{\bar{P} e}{\kappa}\left(-\bar{F}_{0}^{R} \frac{z^{2}}{2}+\left(\bar{F}_{0}^{R}-\bar{f}\right) z+k_{4}\right) \\
c_{2}^{L} & =\frac{\bar{P} e}{\kappa}\left(\frac{k_{5}}{\bar{P} e} z+\frac{\bar{H} \phi k_{1}}{\kappa^{\frac{1}{2}}}\right)-\bar{H}\left(\frac{\bar{P} e}{\kappa}\right)^{3}\left(\frac{\bar{F}_{0}^{L 2} z^{4}}{8}-\frac{\bar{F}_{0}^{L} k_{1} z^{3}}{2 \bar{P} e}+\frac{k_{1}^{2} z^{2}}{2 \bar{P} e^{2}}\right) \\
& -\bar{\eta}^{L}\left(\frac{\bar{P} e}{\kappa}\right)^{2}\left(-\frac{\bar{F}_{0}^{L} z^{4}}{24}+\frac{k_{1} z^{3}}{\bar{P} e 6}\right)-\frac{k_{1} \bar{H} \bar{P} e \phi}{\kappa^{\frac{3}{2}}} e^{-\left(\frac{\kappa}{\epsilon}\right)^{\frac{1}{2}} z} \\
c_{2}^{S} & =\frac{\bar{P} e}{\kappa}\left(k_{6} z+k_{7}\right)-\bar{\eta}^{S}\left(\frac{\bar{P} e}{\kappa}\right)^{2}\left(-\frac{\bar{F}_{0}^{S} z^{4}}{24}+\frac{k_{2} z^{3}}{6}+\frac{k_{3} z^{2}}{2}\right)
\end{aligned}
$$




$$
\begin{aligned}
& -\bar{H}\left(\frac{\bar{P} e}{\kappa}\right)^{3}\left(\frac{\bar{F}_{0}^{S 2} z^{4}}{8}-\frac{\bar{F}_{0}^{S} k_{2} z^{3}}{2}+\frac{\left(k_{2}^{2}-\bar{F}_{0}^{S} k_{3}\right) z^{2}}{2}+k_{2} k_{3} z\right), \\
c_{2}^{R}= & -\bar{H}\left(\frac{\bar{P} e}{\kappa}\right)^{3}\left(\frac{\bar{F}_{0}^{R 2} z^{4}}{8}-\frac{\left(\bar{F}_{0}^{R}-\bar{f}\right) \bar{F}_{0}^{R} z^{3}}{2}\right. \\
& \left.+\frac{\left(\left(\bar{F}_{0}^{R}-\bar{f}\right)^{2}-\bar{F}_{0}^{R} k_{4}\right) z^{2}}{2}+k_{4}\left(\bar{F}_{0}^{R}-\bar{f}\right) z\right) \\
& -\bar{\eta}^{R}\left(\frac{\bar{P} e}{\kappa}\right)^{2}\left(-\frac{\bar{F}_{0}^{R} z^{4}}{24}+\frac{\left(\bar{F}_{0}^{R}-\bar{f}\right) z^{3}}{6}+\frac{k_{4} z^{2}}{2}-\left(\frac{\bar{F}_{0}^{R}}{3}-\frac{\bar{f}}{2}+k_{4}\right) z\right) \\
& +k_{8}-\frac{\bar{H} \bar{P}^{2} \phi \bar{f}}{\kappa^{\frac{3}{2}}} e^{-\left(\frac{\kappa}{\epsilon}\right)^{\frac{1}{2}}(1-z)},
\end{aligned}
$$

The exponential terms of $c_{3}$ are

$$
\begin{aligned}
& c_{3}^{L, e x p}=-\frac{k_{5} \bar{H} \bar{P} e \phi}{\kappa^{\frac{3}{2}}} e^{-\left(\frac{\kappa}{\epsilon}\right)^{\frac{1}{2}} z}-\frac{A_{5} \bar{P} e \phi}{\kappa^{\frac{1}{2}}} e^{-\left(\frac{\kappa}{\epsilon}\right)^{\frac{1}{2}}\left(z_{1}-z\right)}, \\
& c_{3}^{S, e x p}=\frac{A_{6} \bar{P} e \phi}{\kappa^{\frac{1}{2}}} e^{-\left(\frac{\kappa}{\epsilon}\right)^{\frac{1}{2}}\left(z-z_{1}\right)}-\frac{A_{7} \bar{P} e \phi}{\kappa^{\frac{1}{2}}} e^{-\left(\frac{\kappa}{\epsilon}\right)^{\frac{1}{2}}\left(z_{2}-z\right)}, \\
& c_{3}^{R, \exp }=\frac{\bar{P} e^{3} \bar{f} \bar{H}\left(\frac{\bar{F}_{0}^{R}}{2}-\bar{f}+k_{4}\right)}{\kappa^{\frac{7}{2}}}\left(-1+\frac{\bar{H} \bar{P} e}{2}(1-z) \phi\left(\frac{\kappa}{\epsilon}\right)^{\frac{1}{2}}\right) e^{-\left(\frac{\kappa}{\epsilon}\right)^{\frac{1}{2}}(1-z)} \\
& +\frac{A_{8} \bar{P} e \phi}{\kappa^{\frac{1}{2}}} e^{-\left(\frac{\kappa}{\epsilon}\right)^{\frac{1}{2}}\left(z-z_{2}\right)}, \\
& \bar{w}_{0}^{L}=\frac{\bar{H} \bar{P} e}{\kappa}\left(-\bar{F}_{0}^{L} z\right)+\frac{k_{1} \bar{H}}{\kappa}\left(1-e^{-\left(\frac{\kappa}{\epsilon}\right)^{\frac{1}{2}} z}\right), \\
& \bar{w}_{0}^{S}=\frac{\bar{H} \bar{P} e}{\kappa}\left(-\bar{F}_{0}^{S} z+k_{2}\right), \\
& \bar{w}_{0}^{R}=\frac{\bar{H} \bar{P} e}{\kappa}\left(-\bar{F}_{0}^{R} z+\left(\bar{F}_{0}^{R}-\bar{f}\right)\right)+\frac{\bar{H} \bar{P} e \bar{f}}{\kappa} e^{-\left(\frac{\kappa}{\epsilon}\right)^{\frac{1}{2}}(1-z)}, \\
& \bar{w}_{1}^{L}=\frac{\bar{H} k_{5}}{\kappa}-\bar{H}^{2}\left(\frac{\bar{P} e}{\kappa}\right)^{3}\left(\frac{\bar{F}_{0}^{L 2} z^{3}}{2}-\frac{3 \bar{F}_{0}^{L} k_{1} z^{2}}{2 \bar{P} e}+\frac{k_{1}^{2} z}{\bar{P} e^{2}}\right) \\
& -\bar{\eta}^{L} \bar{H}\left(\frac{\bar{P} e}{\kappa}\right)^{2}\left(-\frac{\bar{F}_{0}^{L} z^{3}}{6}+\frac{k_{1} z^{2}}{\bar{P} e 2}\right)-\frac{\bar{H} k_{5}}{\kappa} e^{-\left(\frac{\kappa}{\epsilon}\right)^{\frac{1}{2}} z}+A_{5} e^{-\left(\frac{\kappa}{\epsilon}\right)^{\frac{1}{2}}\left(z_{1}-z\right)}, \\
& \bar{w}_{1}^{S}=\frac{\bar{H} \bar{P} e}{\kappa} k_{6}-\bar{H}^{2}\left(\frac{\bar{P} e}{\kappa}\right)^{3}\left(\frac{\bar{F}_{0}^{S 2} z^{3}}{2}-\frac{3 \bar{F}_{0}^{S} k_{2} z^{2}}{2}+\left(k_{2}^{2}-\bar{F}_{0}^{S} k_{3}\right) z+k_{2} k_{3}\right) \\
& -\bar{\eta}^{S} \bar{H}\left(\frac{\bar{P} e}{\kappa}\right)^{2}\left(-\frac{\bar{F}_{0}^{S} z^{3}}{6}+\frac{k_{2} z^{2}}{2}+k_{3} z\right) \\
& +A_{6} e^{-\left(\frac{\kappa}{\epsilon}\right)^{\frac{1}{2}}\left(z-z_{1}\right)}+A_{7} e^{-\left(\frac{\kappa}{\epsilon}\right)^{\frac{1}{2}}\left(z_{2}-z\right)},
\end{aligned}
$$




$$
\begin{aligned}
\bar{w}_{1}^{R} & =-\bar{H}^{2}\left(\frac{\bar{P} e}{\kappa}\right)^{3}\left(\frac{\bar{F}_{0}^{R 2} z^{3}}{2}-\frac{3\left(\bar{F}_{0}^{R}-\bar{f}\right) \bar{F}_{0}^{R} z^{2}}{2}\right. \\
& \left.+\left(\left(\bar{F}_{0}^{R}-\bar{f}\right)^{2}-\bar{F}_{0}^{R} k_{4}\right) z+k_{4}\left(\bar{F}_{0}^{R}-\bar{f}\right)\right)+A_{8} e^{-\left(\frac{\kappa}{\epsilon}\right)^{\frac{1}{2}}\left(z-z_{2}\right)} \\
& -\bar{\eta}^{R} \bar{H}\left(\frac{\bar{P} e}{\kappa}\right)^{2}\left(-\frac{\bar{F}_{0}^{R} z^{3}}{6}+\frac{\left(\bar{F}_{0}^{R}-\bar{f}\right) z^{2}}{2}+k_{4} z-\left(\frac{\bar{F}_{0}^{R}}{3}-\frac{\bar{f}}{2}+k_{4}\right)\right) \\
& -\bar{H}^{2} \bar{f}\left(\frac{\bar{P} e}{\kappa}\right)^{3}\left(\frac{\bar{F}_{0}^{R}}{2}-\bar{f}+k_{4}\right)\left(1+\frac{\left(\frac{\kappa}{\epsilon}\right)^{\frac{1}{2}}(1-z)}{2}\right) e^{-\left(\frac{\kappa}{\epsilon}\right)^{\frac{1}{2}}(1-z)},
\end{aligned}
$$

The constants $k_{1}$ to $k_{4}$ and $A_{1}$ to $A_{4}$ are given in equation, and $k_{5}, k_{6}, k_{7}$, $k_{8}, A_{5}, A_{6}, A_{7}, A_{8}$ are given by

$$
\begin{aligned}
& A_{5}=\frac{\bar{H} \bar{P} e}{2 \kappa^{\frac{3}{2}}}\left(\bar{F}_{0}^{L}-\bar{F}_{0}^{S}\right), \quad A_{6}=A_{5}, \\
& A_{7}=\frac{\bar{H} \bar{P} e}{2 \kappa^{\frac{3}{2}}}\left(\bar{F}_{0}^{S}-\bar{F}_{0}^{R}\right), \quad A_{7}=A_{8}, \\
& k_{5}=\frac{\bar{\eta}^{L} \bar{P} e^{2} z_{1}^{2}}{2 \kappa}\left(z_{1}\left(\frac{2}{3} \bar{F}_{0}^{L}-\bar{F}_{0}^{S}\right)+z_{2}\left(\bar{F}_{0}^{S}-\bar{F}_{0}^{R}\right)+\bar{F}_{0}^{R}-\bar{f}\right) \\
& +\frac{\left(z_{1}-z_{2}\right) \bar{\eta}^{S} \bar{P} e^{2}}{2 \kappa}\left(-z_{1}^{2} \bar{F}_{0}^{L}+\frac{2}{3} \bar{F}_{0}^{S}\left(z_{1}-z_{2}\right)\left(2 z_{1}+z_{2}\right)\right. \\
& \left.+\bar{F}_{0}^{R}\left(z_{1}+z_{2}\right)\left(z_{2}-1\right)+\bar{f}\left(z_{1}+z_{2}\right)\right) \\
& +\frac{\bar{\eta}^{R} \overline{P e^{2}}\left(z_{2}-1\right)}{2 \kappa}\left(-z_{1}^{2} \bar{F}_{0}^{L}+\bar{F}_{0}^{S}\left(z_{1}+z_{2}\right)\left(z_{1}-z_{2}\right)\right. \\
& \left.+\frac{2}{3} \bar{F}_{0}^{R}\left(2 z_{2}+1\right)\left(z_{2}-1\right)+\bar{f}\left(z_{2}+1\right)\right), \\
& k_{6}=\frac{\bar{\eta}^{S} \bar{P} e z_{2}}{2 \kappa}\left(z_{1}^{2} \bar{F}_{0}^{L}-\frac{1}{3} \bar{F}_{0}^{S}\left(3 z_{1}^{2}-2 z_{2}^{2}\right)-\bar{F}_{0}^{R} z_{2}\left(z_{2}-1\right)-z_{2} \bar{f}\right) \\
& +\frac{\bar{\eta}^{R} \bar{P} e\left(z_{2}-1\right)}{2 \kappa}\left(-z_{1}^{2} \bar{F}_{0}^{L}+\bar{F}_{0}^{S}\left(z_{1}+z_{2}\right)\left(z_{1}-z_{2}\right)\right. \\
& \left.+\frac{2}{3} \bar{F}_{0}^{R}\left(2 z_{2}+1\right)\left(z_{2}-1\right)+\bar{f}\left(z_{2}+1\right)\right), \\
& k_{7}=-\frac{1}{8 \kappa^{2}} \bar{P} e^{2} \bar{H} z_{1}^{4}\left(\bar{F}_{0}^{L}-\bar{F}_{0}^{S}\right)^{2} \\
& +\frac{\bar{H} \bar{P} e \phi}{\kappa^{\frac{1}{2}}}\left(z_{1}\left(\bar{F}_{0}^{L}-\bar{F}_{0}^{S}\right)+z_{2}\left(\bar{F}_{0}^{S}-\bar{F}_{0}^{R}\right)+\bar{F}_{0}^{R}-\bar{f}\right) \\
& +\frac{\bar{\eta}^{L} \bar{P} e z_{1}^{3}}{24 \kappa}\left(z_{1}\left(5 \bar{F}_{0}^{L}-8 \bar{F}_{0}^{S}\right)+8 z_{2}\left(\bar{F}_{0}^{S}-\bar{F}_{0}^{R}\right)+8 \bar{F}_{0}^{R}-8 \bar{f}\right)
\end{aligned}
$$




$$
\begin{aligned}
& -\frac{\bar{\eta}^{S} \bar{P} e z_{1}^{3}}{24 \kappa}\left(z_{1}\left(6 \bar{F}_{0}^{L}-9 \bar{F}_{0}^{S}\right)+8 z_{2}\left(\bar{F}_{0}^{S}-\bar{F}_{0}^{R}\right)+8 \bar{F}_{0}^{R}-8 \bar{f}\right),(\mathrm{C} \\
k_{8}= & -\frac{\bar{P} e^{3} \bar{H}}{8 \kappa^{3}}\left(z_{1}^{2}\left(\bar{F}_{0}^{L}-\bar{F}_{0}^{S}\right)+z_{2}^{2}\left(\bar{F}_{0}^{S}-\bar{F}_{0}^{R}\right)\right)^{2} \\
& +\frac{\bar{H} \bar{P} e^{2} \phi}{\kappa^{\frac{3}{2}}}\left(z_{1}\left(\bar{F}_{0}^{L}-\bar{F}_{0}^{S}\right)+z_{2}\left(\bar{F}_{0}^{S}-\bar{F}_{0}^{R}\right)+\bar{F}_{0}^{R}-\bar{f}\right) \\
& -\frac{\bar{\eta}^{R} \bar{P} e^{2} z_{2}^{2}}{24 \kappa^{2}}\left(6 z_{1}^{2}\left(\bar{F}_{0}^{L}-\bar{F}_{0}^{S}\right)+z_{2}^{2}\left(6 \bar{F}_{0}^{S}-9 \bar{F}_{0}^{R}\right)+8 z_{2}\left(\bar{F}_{0}^{R}-\bar{f}\right)\right) \\
& +\frac{\bar{\eta}^{L} \bar{P} e^{2} z_{1}^{3}}{24 \kappa^{2}}\left(z_{1}\left(5 \bar{F}_{0}^{L}-8 \bar{F}_{0}^{S}\right)+8 z_{2}\left(\bar{F}_{0}^{S}-\bar{F}_{0}^{R}\right)+8 \bar{F}_{0}^{R}-8 \bar{f}\right) \\
& +\frac{\bar{\eta}^{S} \bar{P} e^{2}\left(z_{1}-z_{2}\right)}{24 \kappa^{2}}\left(\bar{F}_{0}^{S}\left(9 z_{1}^{2}+10 z_{1} z_{2}+5 z_{2}^{2}\right)\left(z_{1}-z_{2}\right)\right. \\
& -6 z_{1}^{2} \bar{F}_{0}^{L}\left(z_{1}+z_{2}\right) \\
& \left.+8 \bar{F}_{0}^{R}\left(z_{2}-1\right)\left(z_{1}^{2}+z_{1} z_{2}+z_{2}^{2}\right)+8 \bar{f}\left(z_{1}^{2}+z_{1} z_{2}+z_{2}^{2}\right)\right)
\end{aligned}
$$

where $\bar{F}_{0}^{L}=\bar{\eta}^{L} c_{0}^{L}+\bar{\sigma}^{L}, \quad \bar{F}_{0}^{S}=\bar{\eta}^{S} c_{0}^{S}+\bar{\sigma}^{S}, \quad \bar{F}_{0}^{R}=\bar{\eta}^{R} c_{0}^{R}+\bar{\sigma}^{R}$. 